\title{
Global Existence via Ginzburg-Landau Formalism and Pseudo-Orbits of Ginzburg-Landau Approximations
}

\author{
Guido Schneider \\ Institut für Angewandte Mathematik, Universität Hannover, Welfengarten 1, D-30167 Hannover, \\ Germany
}

Received: 16 July 1993/in revised form: 14 October 1993

\begin{abstract}
The so-called Ginzburg-Landau formalism applies for parabolic systems which are defined on cylindrical domains, which are close to the threshold of instability, and for which the unstable Fourier modes belong to non-zero wave numbers. This formalism allows to describe an attracting set of solutions by a modulation equation, here the Ginzburg-Landau equation. If the coefficient in front of the cubic term of the formally derived Ginzburg-Landau equation has negative real part the method allows to show global existence in time in the original system of all solutions belonging to small initial conditions in $L^{\infty}$. Another aim of this paper is to construct a pseudo-orbit of Ginzburg-Landau approximations which is close to a solution of the original system up to $t=\infty$. We consider here as an example the socalled Kuramoto-Shivashinsky equation to explain the methods, but it applies also to a wide class of other problems, like e.g. hydrodynamical problems or reaction-diffusion equations, too.
\end{abstract}

\section{Introduction and Results}

We consider evolutionary problems over a domain with one unbounded spacedirection close to the threshold of instability. If a spatially homogeneous solution of a dissipative system becomes unstable, a whole band of Fourier modes with positive growth rates appears. In classical bifurcation theory with discrete spectrum the bifurcating solutions can be described by a finite dimensional system (ODE) using center manifold theory (see e.g. [He81]). In our case the spectrum of the linearization at the trivial state is continuous.. Hence new problems appear: First the criticial eigenspace is infinite dimensional and second it cannot be separated from the uncritical part by a spectral gap. Thus, center manifold theory is no longer available. One way to handle such systems is given in [Mi92] leading to PDE's with nonlocal terms. Another way is the so-called Ginzburg-Landau formalism [IMD89] which is based on multiple scaling and on the assumption that the unstable Fourier modes belong to non-zero wave numbers. A formally derived PDE called the Ginzburg-Landau equation takes 
the role of the finite dimensional ODE in center manifold theory. A general set-up for applying the Ginzburg-Landau formalism can be found in [Sch93].

A good model equation for studying the Ginzburg-Landau formalism is the socalled Kuramoto-Shivashinsky equation:

$$
\partial_{t} u=-\left(1+\partial_{x}^{2}\right)^{2} u+\varepsilon^{2} u+u \partial_{x} u=\lambda\left(i \partial_{x}, \varepsilon^{2}\right) u+\varrho\left(i \partial_{x}\right) u^{2}
$$

with $t \geq 0, x \in \mathbb{R}$ and $1 \gg \varepsilon^{2}>0$ the bifurcation parameter. The trivial solution $u \equiv 0$ is unstable, and linearizing at $u \equiv 0$ we find solutions of the form $u(x, t)=e^{\hat{\lambda} t+i k x}$, where $\hat{\lambda}\left(k, \varepsilon^{2}\right)=-\left(1-k^{2}\right)^{2}+\varepsilon^{2}$. We observe that $\hat{\lambda}\left(k, \varepsilon^{2}\right)$ is positive for $k$ close to \pm 1 with height $\mathscr{Q}\left(\varepsilon^{2}\right)$ and width $\mathscr{Q}(\varepsilon)$. Therefore, by inverse Fourier transform we expect that for small $\varepsilon^{2}>0$ there are solutions of (1), which are small modulations in time and in space of the critical modes $e^{ \pm i x}$. Using the scalings $T=\varepsilon^{2} t$ and $X=\varepsilon x$ we suppose that

$$
u(x, t, \varepsilon)=\psi_{\varepsilon}(A)+\mathscr{O}\left(\varepsilon^{3 / 2}\right)=\left[\varepsilon A(X, T) e^{\imath x}+\varepsilon \bar{A}(X, T) e^{-i x}\right]+\mathscr{O}\left(\varepsilon^{3 / 2}\right) .
$$

By a formal calculation we find that the complex valued amplitude $A: \mathbb{R} \times \mathbb{R}^{+} \rightarrow \mathbb{C}$ has to satisfy the Ginzburg-Landau equation

$$
\partial_{T} A(X, T)=A(X, T)+4 \partial_{X}^{2} A(X, T)-(1 / 9) A(X, T)|A(X, T)|^{2} .
$$

Contrary to the spatial periodic case, where center manifold theory applies, the amplitude $A$ depends now also on the slow space variable $X$. This kind of approximation was introduced by Newell and Whitehead (see [NW69]) in 1969 for Bénard's problem (see also (diPES71]). In other hydrodynamic problems, like the Taylor-Couette problem or Poiseuille flow such an approximation is also possible, due to the form of the spectrum. For all these problems the remaining information about the system is contained in the coefficients of the Ginzburg-Landau equation. These are in general complex valued. As a first step in making this formalism rigorous in several papers (see [CE90, KSM92, vH91, Sch92b, Sch93]) the approximation property was shown:

On an Q(1)-time scale of the formally derived Ginzburg-Landau equation (here (2)) the approximation $\psi_{\varepsilon}(A)$ is $\mathscr{G}\left(\varepsilon^{3 / 2}\right)$-close to a solution $u$ of the original problem (here (1)).

The proof for the general case is based on the separation of critical and uncritical Fourier modes and on the fact that the convolution of critical Fourier modes which belong to non-zero wave numbers gives uncritical Fourier modes.

A second step in making this formalism rigorous is the proof of the attractivity of the set of solutions which can be described by the Ginzburg-Landau formalism:

Solutions $u$ to initial conditions of order $\mathscr{O}(\varepsilon)$ develop in such a way that there exists at a time $T_{0} / \varepsilon^{2}$ an initial condition $A_{0}$ of the formally derived GinzburgLandau equation (here (2)), so that the associated approximation $\psi_{\varepsilon}(A)$ possesses the approximation property defined above. We have $T_{0}=\mathscr{O}(1)>0$.

W. Eckhaus has shown this property in [Eck93] for initial conditions whose Fourier transform is in $L^{1} \cap L^{\infty}$. A combination of these two ideas allows to show the global existence principle:

Suppose the coefficient in front of the cubic term of the formally derived GinzburgLandau equation (here (2)) has negative real part. Then all solutions $u$ of the original 
system (here (1)) which belong to initial conditions of order $\mathcal{O}(\varepsilon)$ exist for $t \in[0, \infty)$ and are uniformly bounded for all time.

The idea is as follows: Let $K_{t}$ be the nonlinear evolution operator of the original system in a Banach space $Z$ and $G_{T}$ the nonlinear evolution operator of the associated Ginzburg-Landau equation in a Banach space $Y$. Let $\psi_{\varepsilon}: Y \rightarrow Z$ be the map which maps the solutions of the Ginzburg-Landau equation to the Ginzburg-Landau approximation. For $M \subset Z$ we define $U_{\delta}(M)=\left\{z \in Z \mid \inf _{y \in M}\|y-z\|_{Z}<\delta\right\}$. We show now that a sufficiently large ball $B_{\varepsilon R_{0}}^{Z}$ with radius $\varepsilon R_{0}$ in $Z$ in the original problem is mapped in itself after a certain time. Therefore, all solutions to small initial conditions stay bounded. The attractivity principle we formulate in the following way.

(ATT) There exists $C, \beta, T_{0}, \varepsilon_{0}>0$ and a ball $B_{R_{1}}^{Y}$ with radius $R_{1}$ in $Y$ for the Ginzburg-Landau equation such that for all $0<\varepsilon<\varepsilon_{0}$,

$$
\sup _{u_{0} \in B_{\varepsilon R_{0}}^{Z}} \inf _{A_{0} \in B_{R_{1}}^{Y}}\left\|K_{T_{0} / \varepsilon^{2}}\left(u_{0}\right)-\psi_{\varepsilon}\left(A_{0}\right)\right\|_{Z}<C \varepsilon^{1+\beta}
$$

and

$$
\sup _{t \in\left[0, T_{0} / \varepsilon^{2}\right]} \sup _{u_{0} \in B_{\varepsilon R_{0}}^{Z}}\left\|K_{t}\left(u_{0}\right)\right\|_{Z} \leq C \varepsilon .
$$

The approximation property is formulated as

(APP) For all $T_{1}, d>0$ there exists $C, \varepsilon_{0}>0$ such that for all $0<\varepsilon<\varepsilon_{0}$ the following holds. Let $A_{0} \in B_{R_{1}}^{Y}$ and $u_{0} \in Z$ with $\left\|u_{0}-\psi_{\varepsilon}\left(A_{0}\right)\right\|_{Z} \leq d \varepsilon^{1+\beta}$, then

$$
\sup _{0 \leq t \leq T_{1} / \varepsilon^{2}}\left\|K_{t}\left(u_{0}\right)-\psi_{\varepsilon}\left(G_{\varepsilon^{2} t}\left(A_{0}\right)\right)\right\|_{Z} \leq C \varepsilon^{1+\beta} .
$$

We assume that the real part of the cubic coefficient is negative. So we expect (GL) There exists a ball $B_{R_{3}}^{Y}$ in $Y$ such that for all $R_{2}>0$ there is a $T_{3}>0$ with

$$
G_{T_{3}}\left(B_{R_{2}}^{Y}\right) \subset B_{R_{3}}^{Y} \quad \text { and } \bigcup_{T \in\left[0, T_{3}\right]} G_{T}\left(B_{R_{2}}^{Y}\right) \quad \text { is a bounded set. }
$$

The space $Y$ has to be chosen such that (GL) is valid. So we cannot take the space used in [Eck93]. It remains to combine these principles. We choose $R_{0}$ independent of $\varepsilon$, so large that $\psi_{\varepsilon}\left(B_{R_{3}}^{Y}\right)$ is contained in $B_{\varepsilon R_{0} / 2}^{Z}$ for all $0<\varepsilon<\varepsilon_{0}$. Then by (ATT) $M_{1}=K_{T_{0} / \varepsilon^{2}}\left(B_{\varepsilon R_{0}}^{Z}\right) \subset U_{C \varepsilon^{1+\beta}}\left(\psi_{\varepsilon}\left(B_{R_{1}}^{Y}\right)\right)$. By (APP) and (GL) follows that

$$
K_{T_{3} / \varepsilon^{2}}\left(M_{1}\right) \subset U_{C \varepsilon^{1+\beta}}\left(\psi_{\varepsilon}\left(G_{T_{3}}\left(B_{R_{1}}^{Y}\right)\right)\right) \subset U_{C \varepsilon^{1+\beta}}\left(\psi_{\varepsilon}\left(B_{R_{3}}^{Y}\right)\right) \subset B_{\varepsilon R_{0}}^{Z}
$$

for sufficiently small $\varepsilon$. We have demonstrated:

Theorem 1. If (ATT), (APP), and (GL) are valid, then there exists $T_{0}, T_{3}, \varepsilon_{0}>0$ such that for all $0<\varepsilon<\varepsilon_{0}$ and $R_{0}$ sufficiently large $K_{\left(T_{0}+T_{3}\right) / \varepsilon^{2}}\left(B_{\varepsilon R_{0}}^{Z}\right) \subset B_{\varepsilon R_{0}}^{Z}$. Therefore, solutions $K_{t}\left(u_{0}\right)$ with initial conditions $u_{0}$ in $B_{\varepsilon R_{0}}^{Z}$ stay bounded and exist for all time.

In the third section we give some versions of (ATT), (APP) and (GL) for the Kuramoto-Shivashinsky equation (1). Doing this we have shown the global existence principle for the Kuramoto-Shivashinsky equation (1). To write this down, we recall 
the functional analytic set-up of [Sch93]: The spaces $H_{l, u}^{n}$ are introduced with norm $\|u\|_{H_{l, u}^{n}(\mathbb{R}, \mathbb{C})}=\sup _{m \in Z}\|u\|_{H^{n}((m, m+2), \mathbb{C})}$. Note that $L^{\infty} \subset L_{l, u}^{2}=H_{l, u}^{0}$. Hence, we can treat fronts and spatially quasiperiodic solutions. Moreover, these spaces allow to handle hydrodynamical problems, too. In these spaces our global existence result is given by

Theorem 2. There exists $\varepsilon_{0}, C, R_{0}>0$, such that for all $0<\varepsilon<\varepsilon_{0}$ and all solutions $K_{t}\left(u_{0}\right)$ of $(1)$ with $\left\|u_{0}\right\|_{H_{l, u}^{4}(\mathbb{R}, \mathbb{R})} \leq R_{0} \varepsilon$ the estimate $\sup _{t \in[0, \infty)}\left\|K_{t}\left(u_{0}\right)\right\|_{H_{l, u}^{4}(\mathbb{R}, \mathbb{R})}<$ $C \varepsilon<\infty$ is valid.

Now we come to the second aim of this paper. For every time interval $\left[\left(T_{0}+\right.\right.$ $\left.\left.n T_{1}\right) / \varepsilon^{2},\left(T_{0}+(n+1) T_{1}\right) / \varepsilon^{2}\right],(n \in \mathbb{N})$, there exists an initial condition $A_{n+1} \in H_{l, u}^{1}$ of the Ginzburg-Landau equation such that $K_{t}\left(u_{0}\right)$ and $\psi_{\varepsilon}\left(G_{\varepsilon^{2} t-\left(T_{0}+n T_{1}\right)}\left(A_{n+1}\right)\right)$ are nearby on this time interval. This can be shown by using (APP) and (ATT) parallel. Obviously the jumps from one approximation to another at a time $t=\left(T_{0}+n T_{1}\right) / \varepsilon^{2}$ are small. A delicate problem is to show that the jumps of the associated solutions of the Ginzburg-Landau equation are small, too. The space we take to estimate these jumps is $H_{l, u}^{1}$ and not only $L_{l, u}^{2}$. For initial conditions $A_{0} \in H_{l, u}^{1}$ we can show the approximation property (APP). Moreover, the solutions depend continuously on the initial conditions. We show

Theorem 3. There exists $T_{0}>0$ such that for all $T_{1}>0$ and all initial conditions $u_{0} \in H_{l, u}^{4}(\mathbb{R}, \mathbb{R})$ for (1) with $\left\|u_{0}\right\|_{H_{l, u}^{4}(\mathbb{R}, \mathbb{R})}<R_{0} \varepsilon$ the following holds. There exists a sequence $\left(A_{n}\right)_{n \in \mathbb{N}}$ of initial conditions $A_{n} \in H_{l, u}^{1}$ for the Ginzburg-Landau equation (2) and there are $\varepsilon_{0}, C>0$ only depending on $R_{0}$ and $T_{1}$ such that for all $0<\varepsilon<\varepsilon_{0}$ the pseudo-orbit

$$
\psi=\psi(t)=\left\{\psi_{\varepsilon}\left(G_{\varepsilon^{2} t-\left(n T_{1}+T_{0}\right)}\left(A_{n+1}\right)\right)\right\}
$$

for

$$
t \in\left[\left(T_{0}+n T_{1}\right) / \varepsilon^{2},\left(T_{0}+(n+1) T_{1}\right) / \varepsilon^{2}\right], n \in \mathbb{N}
$$

fulfills

$$
\sup _{t \geq T_{0} / \varepsilon^{2}}\left\|K_{t}\left(u_{0}\right)-\psi(t)\right\|_{H_{l, u}^{4}} \leq C \varepsilon^{5 / 4}
$$

Moreover,

$$
\sup _{n}\left\|G_{T_{1}} A_{n}-A_{n+1}\right\|_{H_{l, u}^{1}} \leq C \varepsilon^{1 / 4} .
$$

This theorem allows to follow the attracting set in the original system by pseudo-orbits of Ginzburg-Landau approximations for all times. We hope that this theorem allows to draw conclusions about the relations between the attractor of the Ginzburg-Landau equation and the attractor of the original problem.

To explain the methods we study the Kuramoto-Shivashinsky equation as a model equation, but nevertheless, these methods developed here can be generalized to a wide class of other problems, like hydrodynamical problems or reaction-diffusion equations, too. This will be done in a forthcoming paper.

The plan of this paper is as follows. In the next section we present some prerequisites, like the functional analytic set-up, the spaces and the so-called multiplier theory. Also, it is shown that the Ginzburg-Landau equation possesses an attracting ball in $H_{l, u}^{1}$. The third section contains the proofs of (APP), (ATT), Theorem 2, and 
Theorem 3. The last section contains a discussion about possible generalizations and applications of the result.

Throughout this paper many different constants are uniformly denoted by $C$.

\section{Prerequisites}

\subsection{The Functional Analytic Set-Up and Mode Filters}

In this section we give the functional analytic set-up to show the above theorems. One difficulty for unbounded domains is the choice of a good space in which the solutions should be. It should include all interesting solutions, like fronts or spatially periodic solutions. It should make Fourier transform available, since Ginzburg-Landau formalism depends heavily on it, and for more complicated problems it should allow to use the analysis of the in general well known spatially periodic case. To our opinion the functional analytic set-up founded in [Sch93] is a good choice. So we recall and extend this set-up in the following.

Fourier transform is defined for tempered distributions $u$ with help of testfunctions and is denoted by $\hat{u}$ or $\mathscr{F} u$. The Fourier transform of an integrable function $u$ is given by $\frac{1}{2 \pi} \int e^{i k x} u(x) d x$. As usually the space $L^{2}(\mathbb{R}, \mathbb{C})$ denotes the space of squareintegrable functions. We define the space $H^{s}(\mathbb{R}, \mathbb{C})$ for $s \geq 0$ as the subspace of $L^{2}(\mathbb{R}, \mathbb{C})$ for which the norm

$$
\|u\|_{H^{s}(\mathbb{R}, \mathbb{C})}^{2}=\int_{\mathbb{R}}|\mathscr{F} u|^{2}\left(1+k^{2}\right)^{s} d k
$$

is finite. This space coincides for $s \in \mathbb{N}$ with the usual definition of Sobolev spaces (see [LM72]). Moreover, the space of $n$-times continuously differentiable functions with bounded derivatives is denoted by $C_{b}^{n}$. It is equipped with the natural norm.

To define the spaces we want to work in, let $\chi_{n}=\chi(x-n)$ be a smooth, periodic partition of unity where $\chi$ has compact support $[-1,1]$. We define now the space

$$
H_{l, u}^{s}(\mathbb{R}, \mathbb{C})=\left\{u: \mathbb{R} \rightarrow \mathbb{C} \mid\|u\|_{H_{l, u}^{s}(\mathbb{R}, \mathbb{C})}=\sup _{n \in Z}\left\|u \chi_{n}\right\|_{H^{s}(\mathbb{R}, \mathbb{C})}<\infty\right\} .
$$

We write $L_{l, u}^{2}(\mathbb{R}, \mathbb{C})$ instead of $H_{l, u}^{0}(\mathbb{R}, \mathbb{C})$ and remark that $L^{\infty}(\mathbb{R}, \mathbb{C}) \subset L_{l, u}^{2}(\mathbb{R}, \mathbb{C})$. Therefore, these spaces have the above demanded property of containing all interesting solutions. The indices $l, u$ stand for locally and uniformly.

The second demanded property of making Fourier transform available is fulfilled with the help of the so-called multiplier theory. To define operators in $H_{l, u}^{s}(\mathbb{R}, \mathbb{C})$ we cite some theorems of [Sch93]. We call operators multipliers if they are defined by multiplying the Fourier transform $\hat{u}=\mathscr{F} u$ by a function $\hat{M} \in L^{\infty}(\mathbb{R}, \mathbb{C})$. Using the following lemma allows us to define operators in the physical space by $M: u \mapsto \mathscr{F}^{-1}(\hat{M} \mathscr{F} u)$.

Lemma 4. Let $q, s \geq 0$ and $k \mapsto\left(1+k^{2}\right)^{(s-q) / 2} \hat{M}(k) \in C_{b}^{2}(\mathbb{R}, \mathbb{C})$. Then $M$ : $H_{l ; u}^{q}(\mathbb{R}, \mathbb{C}) \rightarrow H_{l ; u}^{s}(\mathbb{R}, \mathbb{C})$ is well defined with the estimate

$$
\begin{aligned}
\|M u\|_{H_{l, u}^{s}(\mathbb{R}, \mathbb{C})} \leq & C(q, s) \min \left\{\left\|k \mapsto\left(1+|k|^{2}\right)^{(s-q) / 2} \hat{M}(k)\right\|_{C_{b}^{2}(\mathbb{R}, \mathbb{C})},\right. \\
& \left.\int\left|\int \hat{M}(k) e^{i k x}\left(1+|k|^{2}\right)^{(s-q) / 2} d k\right| d x\right\}\|u\|_{H_{l, u}^{q}(\mathbb{R}, \mathbb{C})},
\end{aligned}
$$

where $C(q, s)$ does not depend on $\hat{M}$. 
An operator $M$ defined in this way is translation invariant, i.e. $\tau_{h} M=M \tau_{h}$, where $\tau_{h} u(y)=u(y+h)$ for all $h \in \mathbb{R}$. Multipliers in Fourier space are denoted with a $\hat{\bullet}$, in physical space without $\hat{\imath}$.

We use this lemma to define special multipliers which allows us to separate critical from uncritical Fourier modes, but we remark that a differential operator or an analytic semigroup can also be interpreted as a multiplier. To extract modes in Fourier space we use an even cut-off function $\hat{\phi}_{0} \in C_{0}^{\infty}$ which is defined by

$$
\hat{\phi}_{0}(k)= \begin{cases}1 & \text { for } k \in[-1 / 6,1 / 6], \\ 0 & \text { for } k \notin[-1 / 3,1 / 3], \\ \in[0,1] & \text { else. }\end{cases}
$$

According to Lemma 4 we associate to $\hat{\phi}_{0}$ an operator which extracts the Fourier modes belonging to wave numbers in $[-1 / 3,1 / 3]$. To extract the critical modes we define $\hat{E}_{1}(k)=\hat{\phi}_{0}(k-1), \hat{E}_{-1}(k)=\hat{\phi}_{0}(k+1)$ and $E_{c}=E_{1}+E_{-1}$. We call these operators mode filters. Since the $E_{j}$ are no projections we need auxiliary operators defined by $\hat{E}_{1}^{h}(k)=\hat{\phi}_{0}((k-1) / 2), \hat{E}_{-1}^{h}(k)=\hat{\phi}_{0}((k+1) / 2)$ and $E_{c}^{h}=E_{1}^{h}+E_{-1}^{h}$. We have for example $E_{c} E_{c}^{h}=E_{c}$. For the uncritical modes we define $E_{s}=$ Id. $-E_{c}$ and $\hat{E}_{s}^{h}(k)=1-\hat{\phi}_{0}(2(k+1))-\hat{\phi}_{0}(2(k-1))$. Using Lemma 4 it is easily seen that $E_{j} u \in H_{l, u}^{s}$ for all $s \geq 0$ if $u \in L_{l, u}^{2}$.

To deal with the slow spatial scale $X=\varepsilon x$ we need a scaling operator $S_{\varepsilon}$ defined by $\left(S_{\varepsilon} u\right)(x)=u(\varepsilon x)$. With help of this operator we define the scaled spaces $H_{l, u}^{s, \varepsilon}$ by $u \in H_{l, u}^{s, \varepsilon}(\mathbb{R}, \mathbb{C}) \Leftrightarrow S_{1 / \varepsilon} u \in H_{l, u}^{s}(\mathbb{R}, \mathbb{C})$ with the norm $\|u\|_{H_{l, u}^{s, \varepsilon}(\mathbb{R}, \mathbb{C})}:=$ $\left\|S_{1 / \varepsilon} u\right\|_{H_{l, u}^{s}(\mathbb{R}, \mathbb{C})}$. Obviously $u \in H_{l, u}^{s}$ gives $S_{\varepsilon} u \in H_{l, u}^{s, \varepsilon}(\mathbb{R}, \mathbb{C})$. Scaling a multiplier leads to

$$
S_{1 / \varepsilon} M u=\varepsilon \mathscr{F}^{-1} S_{\varepsilon}(\hat{M} \mathscr{F} u)=\mathscr{F}^{-1}\left(S_{\varepsilon} \hat{M}\right) \mathscr{F} S_{1 / \varepsilon} u
$$

which is easily seen by applying test functions. Moreover, we define $V_{k}: H_{l, u}^{s} \rightarrow H_{l, u}^{s}$, $u(x) \mapsto u(x) e^{i k x}$. Applying the above lemma on the new multiplier $\mathscr{F}^{-1}\left(S_{\varepsilon} \hat{M}\right) \mathscr{F}$ we get

Lemma 5. Let $q, s \geq 0$ and $k \mapsto\left(1+|k|^{2}\right)^{(s-q) / 2} \hat{M}(k) \in C_{b}^{2}(\mathbb{R}, \mathbb{C})$. Then $M$ : $H_{l ; u}^{q, \varepsilon}(\mathbb{R}, \mathbb{C}) \rightarrow H_{l ; u}^{s, \varepsilon}(\mathbb{R}, \mathbb{C})$ is well defined with the estimate

$$
\begin{aligned}
& \left\|V_{-k_{0}}\left(M\left(V_{k_{0}} u\right)\right)\right\|_{H_{l, u}^{s, \varepsilon}(\mathbb{R}, \mathbb{C})} \\
& \quad \leq C(q, s)\left\|k \mapsto\left(1+|k|^{2}\right)^{(s-q) / 2} \hat{M}\left(\varepsilon k+k_{0}\right)\right\|_{C_{b}^{2}(\mathbb{R}, \mathbb{C})}\|u\|_{H_{l, u}^{q, \varepsilon}(\mathbb{R}, \mathbb{C})}
\end{aligned}
$$

where $C(q, s)$ does not depend on $\hat{M}$ and $\varepsilon$.

Remark 6. If additionally $\hat{M}\left(k+k_{0}\right)=\mathscr{Q}\left(k^{s}\right)$ for $k \rightarrow 0$ and $s \leq m$. Then

$$
\left\|k \mapsto\left(1+|k|^{2}\right)^{-m / 2} \hat{M}\left(\varepsilon k+k_{0}\right)\right\|_{C_{b}^{2}(\mathbb{R}, \mathbb{C})}=\mathscr{Q}\left(\varepsilon^{s}\right) .
$$

This follows by simple calculations.

Obviously we have to estimate a scaled function by its unscaled version and so we recall [Sch93] that we have only $\left\|S_{\varepsilon} u\right\|_{L_{l, u}^{2}(\mathbb{R}, \mathbb{C})} \leq C \max \left(\varepsilon^{-1 / 2}, 1\right)\|u\|_{L_{l, u}^{2}(\mathbb{R}, \mathbb{C})}$. Hence, for $0<\varepsilon \leq 1$,

$$
\frac{1}{C}\|u\|_{L_{l, u}^{2, \varepsilon}(\mathbb{R}, \mathbb{C})} \leq\|u\|_{L_{l, u}^{2}(\mathbb{R}, \mathbb{C})} \leq C \max \left(\varepsilon^{-1 / 2}, 1\right)\|u\|_{L_{l, u}^{2, \varepsilon}(\mathbb{R}, \mathbb{C})} .
$$


Since $H_{l, u}^{s}$ can be imbedded in $L^{\infty}$ for $s>1 / 2$, we get easily $\left\|S_{\varepsilon} u\right\|_{L_{l, u}^{2}(\mathbb{R}, \mathbb{C})} \leq$ $C\|u\|_{H_{l, u}^{s}(\mathbb{R}, \mathbb{C})}$.

Example. Take $A \in H_{l, u}^{1}$, then

$$
\left\|E_{1}^{h} S_{\varepsilon} A\right\|_{H_{l, u}^{n}} \leq C\left\|k \mapsto \hat{E}_{1}^{h}(k)\left(1+|k|^{2}\right)^{+n / 2}\right\|_{C_{b}^{2}}\left\|S_{\varepsilon} A\right\|_{L_{l, u}^{2}} \leq C\|A\|_{H_{l, u}^{1}} .
$$

Remark. In Lemma 4 we gave two different estimates for the operator norm. This is necessary since

$$
\begin{aligned}
\left\|e^{-k^{2} t}\right\|_{C_{b}^{2}} & =\sup _{k}\left|e^{-k^{2} t}\right|+\sup _{k}\left|(-2 k t) e^{-k^{2} t}\right|+\sup _{k}\left|\left(-2 t+4 k^{2} t^{2}\right) e^{-k^{2} t}\right| \\
& \leq C \max (1, \sqrt{t}, t) .
\end{aligned}
$$

On the long time scale $1 / \varepsilon^{2}$ this is of order $\mathscr{Q}\left(1 / \varepsilon^{2}\right)$. Remark that

$$
\int\left|\int e^{-k^{2} t} e^{i k x} d k\right| d x \leq C \int\left|\int e^{-s^{2}} e^{i l s} d s\right| d l \leq C .
$$

Therefore we can estimate the semigroup $e^{\mu t}$ generated by the multiplier $\hat{\mu}=-k^{2}$ for all $t>0$ by $\left\|e^{\mu t}\right\|_{\mathscr{L}\left(H_{l, u}^{4}, H_{l, u}^{4}\right)} \leq C$. This problem will not appear on the long time scale if we apply the semigroup on scaled functions (estimate $\left\|e^{-\varepsilon^{2} k^{2} t}\right\|_{C_{b}^{2}}$ ), since the diffusion happens on longer time scales. Moreover, if the semigroup is exponentially damped (estimate $\left\|e^{-\sigma t} e^{-k^{2} t}\right\|_{C_{b}^{2}}$ ) we again obtain good results.

\subsection{The Ginzburg-Landau Equation}

In this section we will show that the Ginzburg-Landau equation possesses an exponentially attracting ball in $H_{l, u}^{1}(\mathbb{R}, \mathbb{C})$. To show this calculations made in [CE90] were used. They can be used, since the norm $\|u\|_{W}^{2}=\sup (u, u)_{h_{a}}$ with weight function $h_{a}(x)=1 /\left(1+(x-a)^{2}\right)$ and inner product $(u, v)_{h_{a}}=\int^{a} u \bar{v} h_{a}^{2} d x$ is equivalent to the above defined norm in $L_{l, u}^{2}(\mathbb{R}, \mathbb{C})$. On one hand we have

$$
\|u\|_{W}=\sup _{a}\left(\int|u|^{2} h_{a}^{2} d x\right)^{1 / 2} \geq C \sup _{n}\left\|u \chi_{n}\right\|_{L^{2}(\mathbb{R}, \mathbb{C})}=C\|u\|_{L_{l, u}^{2}(\mathbb{R}, \mathbb{C})} .
$$

On the other hand

$$
\begin{aligned}
\|u\|_{W} & =\sup _{a}\left(\int|u|^{2} h_{a}^{2} d x\right)^{1 / 2} \leq C \sup _{a}\left(\sum_{n} \int|u|^{2} h_{a}^{2} \chi_{n}^{2} d x\right)^{1 / 2} \\
& \leq C \sup _{a}\left(\sum_{n} \sup _{y \in[n, n+2]} h_{a}^{2}\right) \sup _{n}\left(\int|u|^{2} \chi_{n}^{2} d x\right)^{1 / 2} \\
& \leq C\|u\|_{L_{l, u}^{2}(\mathbb{R}, \mathbb{C})}
\end{aligned}
$$


and so the equivalence of the norm follows. Let $A$ now be a solution of the GinzburgLandau equation. Define $S_{a}=\int A \bar{A} h_{a}^{2} d x$ and $\tilde{S}_{a}=\int A_{x} \bar{A}_{x} h_{a}^{2} d x$. From the calculations made in [CE90] one can conclude that

$$
S_{a}(T) \leq\left. e^{-2 T} S_{a}\right|_{T=0}+C \quad \text { and } \quad \tilde{S}_{a}(T) \leq\left. e^{-2 T} \tilde{S}_{a}\right|_{T=0}+C
$$

where the constant $C$ does not depend on $a$. Taking now on both sides the supremum we can summarize

Theorem 7. Let A be a solution of the Ginzburg-Landau equation (2) then there exists a constant $C_{b}$, such that

$$
\|A(T)\|_{H_{l, u}^{1}(\mathbb{R}, \mathbb{C})} \leq C_{b}+e^{-T}\|A(0)\|_{H_{l, u}^{1}(\mathbb{R}, \mathbb{C})} .
$$

To show the approximation property for initial conditions for the Ginzburg-Landau equation in $H_{l, u}^{1}$ we have to make use of the smoothing properties of the GinzburgLandau equation. Using the variation of constants formula to transform the differential equation into an integral equation we can conclude

Lemma 8. Let $A \in C\left([0,1], H_{l, u}^{1}(\mathbb{R}, \mathbb{C})\right)$ be a solution of the Ginzburg-Landau equation, then $\|A(T)\|_{H_{l, u}^{1+s}} \leq C_{s} / T^{s / 2}+C_{s}$, where $C_{s}$ is a constant depending on $0 \leq s<2$

Remark 9. Theorem 7 is also true for the complex Ginzburg-Landau equation

$$
\partial_{T} A=c_{1} A+c_{2} \partial_{X}^{2} A+c_{3} A|A|^{2}, \quad\left(c_{\imath} \in \mathbb{C}\right),
$$

if the coefficient $c_{3}$ has negative real part. To get (4) Lemma 4.2 of [CE90] is needed to control the linear part of (5). It applies also in this situation since it is sufficient to have $\Re c_{2}>0$, where $\Re c_{2}$ denotes the real part of $c_{2}$, as it is assumed in [Ho85] Theorem 18.1.14-15 which is used to prove this lemma. We have $\Re c_{2}>0$ since the Ginzburg-Landau formalism only applies in these situations of supercritical bifurcations.

\section{The Proof}

To show the main principles of the introduction and the existence of a pseudo-orbit as asserted in Theorem 3 three lemmas are given. The first lemma allows to choose an initial condition of the Ginzburg-Landau equation and to start the approximation process. The second lemma brings us into a situation which allows to copy more or less the proof of the approximation theorem of [Sch92b]. The third lemma shows the approximation property. The differences between this lemma and previous works are explained below. By a combination of these lemmas the assertions will follow.

Before we start we recall that we consider real systems. Therefore it is sufficient to estimate either $E_{1}^{h} u$ or $E_{-1}^{h} u$. The lemmas are proved in a succeeding section.

\subsection{Statement of the Main Lemmas and Proof of Theorem 2}

By previous works it is known that it is essential to separate the critical modes from the uncritical modes of a solution. The critical modes are strongly related to the 
solutions of the Ginzburg-Landau equation. In a first step we show that solutions develop in such a way that the critical part can be taken as the initial condition for the Ginzburg-Landau equation.

Lemma 10. Let $u_{0}$ be an initial condition for (1) with $\left\|u_{0}\right\|_{H_{l, u}^{4}} \leq R_{0} \varepsilon$. Then there exists $1>T_{0}, \varepsilon_{0}>0$ such that for all $0<\varepsilon<\varepsilon_{0}$ the following holds:

a) There exists $C>0$ only depending on $R_{0}$ such that

$$
\sup _{0 \leq t \leq T_{0} / \varepsilon^{2}} \sup _{\left\|u_{0}\right\|_{H_{l, u}^{4}} \leq R_{0} \varepsilon}\left\|K_{t}\left(u_{0}\right)\right\|_{H_{l, u}^{4}}<C .
$$

b) For $t=T_{0} / \varepsilon^{2}$ the solution $K_{t}\left(u_{0}\right)$ can be written as

$$
K_{T_{0} / \varepsilon^{2}}\left(u_{0}\right)=\varepsilon w_{c}+\varepsilon^{2} w_{s}
$$

with $w_{c}=E_{c}^{h} w_{c}$ and $w_{s}=E_{s}^{h} w_{s}$. There exists constants $C_{1}, C_{2}$ only depending on $R_{0}$ and not on $\varepsilon$ or $u_{0}$ itself such that $\left\|V_{-1} E_{1}^{h} w_{c}\right\|_{H_{l, u}^{1, \varepsilon}} \leq C_{1}$ and $\left\|w_{s}\right\|_{H_{l, u}^{4}} \leq C_{2}$.

This lemma allows to take $A_{1}=S_{1 / \varepsilon} V_{-1} E_{1}^{h} w_{c} \in H_{l, u}^{1}$ as the first initial condition of the Ginzburg-Landau equation (2). For a technical reason the mapping $\psi_{\varepsilon}$ which maps $A \in H_{l, u}^{1}$ to the approximation $\psi_{\varepsilon}(A)$ is not given by $u_{\psi}(A)=$ $\varepsilon A(X, T) e^{\imath x}+$ c.c. The chosen mapping $\psi_{\varepsilon}$ is close to $u_{\psi}$, but has much better properties. First, $\psi_{\varepsilon}(A)$ is highly regular, and second, it makes the so-called residuum

$$
\operatorname{Res}(\psi)=-\partial_{t} \psi+\lambda \psi+\varrho \psi^{2}
$$

small. This residuum appears as inhomogenity in the equations for the error $R$ and contains all remaining terms which do not drop out after inserting $\psi_{\varepsilon}(A)$ in (1), if $A$ is solution of (2).

In a classical way the order of the residuum can be made small by taking more terms into the approximation. By applying appropriate mode filters on this extended approximation we obtain a highly regular approximation $\psi_{\varepsilon}(A)$ nearby $u_{\psi}(A)$.

To derive formally the Ginzburg-Landau equation we insert as normally

$$
\tilde{\psi}=\left(\varepsilon A_{1}^{0}\left(\varepsilon x, \varepsilon^{2} t\right) e^{i x}+\varepsilon^{2} A_{2}^{0}\left(\varepsilon x, \varepsilon^{2} t\right) e^{2 i x}+\text { c.c. }\right)+\varepsilon^{2} A_{0}^{0}\left(\varepsilon x, \varepsilon^{2} t\right)
$$

in (1) and get in lowest order the modulation equations

$$
\begin{aligned}
\partial_{T} A_{1}^{0} & =\left(1+4 \partial_{x}^{2}\right) A_{1}^{0}+i A_{-1}^{0} A_{2}^{0}+i A_{0}^{0} A_{1}^{0}, \\
0 & =-9 A_{2}^{0}+i A_{1}^{0} A_{1}^{0}, \\
0 & =-A_{0}^{0} .
\end{aligned}
$$

Upon elimination of $A_{ \pm 2}^{0}$ we arrive at the Ginzburg-Landau equation (2). Formally in the coefficient in front of $e^{i x}$ all terms of order $\mathscr{Q}\left(\varepsilon^{3}\right)$ and for 1 and $e^{2 i x}$ all terms of order $\mathscr{Q}\left(\varepsilon^{2}\right)$ have vanished. Now Lemma 5 allows us to smooth $\tilde{\psi}$ without changing it very much. Therefore we define another mode filter $E$ defined by the multiplier $k \mapsto \hat{\phi}_{0}(3 k)$ in Fourier space. Moreover, we define $E_{0}=E S_{\varepsilon}$. We modify the formal approximation by applying this mode-filter and take finally

$$
\begin{aligned}
\psi_{\varepsilon}\left(A_{1}^{0}\right) & =\varepsilon \psi_{c}\left(A_{1}^{0}\right)+\varepsilon^{2} \psi_{s}\left(A_{1}^{0}\right), \\
\varepsilon \psi_{c}\left(A_{1}^{0}\right) & =\varepsilon\left(E_{0} A_{1}^{0}\left(\varepsilon^{2} t\right)\right) e^{i x}+\varepsilon\left(E_{0} A_{-1}^{0}\left(\varepsilon^{2} t\right)\right) e^{-i x}, \\
\varepsilon^{2} \psi_{s}\left(A_{1}^{0}\right) & =\varepsilon^{2}\left(E_{0} A_{2}^{0}\left(\varepsilon^{2} t\right)\right) e^{2 i x}+\varepsilon^{2}\left(E_{0} A_{-2}^{0}\left(\varepsilon^{2} t\right)\right) e^{-2 i x},
\end{aligned}
$$


where $A_{2}^{0}=(i / 9)\left(A_{1}^{0}\right)^{2}$. Note that $E_{s}^{h} \psi_{c}=0$ and $E_{c}^{h} \psi_{s}=0$. Using Lemma 5 and (3) we have

$$
\sup _{t \in\left[0, T_{0} / \varepsilon^{2}\right]}\left\|\psi_{\varepsilon}\left(A_{1}^{0}\right)-u_{\psi}\left(A_{1}^{0}\right)\right\|_{L_{l, u}^{2}} \leq \mathcal{Q}\left(\varepsilon^{3 / 2}\right),
$$

if $A_{1}^{0} \in H_{l, u}^{1}$. Moreover, $\psi_{c}\left(A_{1}^{0}\right)$ is of order $\mathcal{Q}(1)$ in $H_{l, u}^{1, \varepsilon} \cap H_{l, u}^{n}$ and $\psi_{s}\left(A_{1}^{0}\right)$ is of order $\mathscr{O}(1)$ in $H_{l, u}^{n}$ for all $n \in \mathbb{N}$. This follows by applying Lemma 4 to $E$.

Let $A_{0} \in H_{l, u}^{1}$ be an initial condition for (2). The error made by the approximation $\psi_{\varepsilon}\left(G_{\varepsilon^{2} t}\left(A_{0}\right)\right)$ of a solution $u$ is given by

$$
R(t)=K_{t}\left(\left.u\right|_{t=T_{0} / \varepsilon^{2}}\right)-\psi_{\varepsilon}\left(G_{\varepsilon^{2} t}\left(A_{0}\right)\right)
$$

It is estimated as solution of the differential equation $\partial_{t} R=F(R, t)$ it fulfill. We separate $R=R_{c}(t)+R_{s}(t)$, where $R_{c}$ and $R_{s}$ are defined to be the solutions of

$$
\begin{aligned}
& \partial_{t} R_{c}=E_{c} F\left(R_{c}+R_{s}, t\right), \\
& \partial_{t} R_{s}=E_{s} F\left(R_{c}+R_{s}, t\right) .
\end{aligned}
$$

For $A_{0}=A_{1}$ the initial conditions are given by $R_{s}(0)=\varepsilon^{2} w_{s}-\varepsilon^{2} \psi_{s}\left(A_{1}\right)$ and by $R_{c}(0)=\varepsilon V_{1}(1-E) V_{-1} E_{1}^{h} w_{c}+$ c.c. Applying Lemma 4 and Lemma 5 we can estimate

$$
\begin{aligned}
\left\|R_{c}(0)\right\|_{H_{l, u}^{4}} \leq & C\left\|R_{c}(0)\right\|_{L_{l, u}^{2}} \leq C \varepsilon^{-1 / 2}\left\|V_{-1} E_{1}^{h} R_{c}(0)\right\|_{L_{l, u}^{2, \varepsilon}} \\
\leq & \left.C \varepsilon^{-1 / 2} \| k \mapsto\left(1+k^{2}\right)^{-1 / 2}(1-\hat{E}(\varepsilon k)) \hat{E}_{1}^{h}(1+\varepsilon k)\right) \|_{C_{b}^{2}} \\
& \times\left\|\varepsilon V_{-1} E_{1}^{h} w_{c}\right\|_{H_{l, u}^{1, \varepsilon}} \\
\leq & C \varepsilon^{3 / 2}\left\|\varepsilon V_{-1} E_{1}^{h} w_{c}\right\|_{H_{l, u}^{1, \varepsilon}} \leq \mathscr{O}\left(\varepsilon^{3 / 2}\right) .
\end{aligned}
$$

In general $R_{s}(t)=E_{s} R(t)$ and $R_{c}(t)=E_{c} R(t)$ is not true for $t>0$, since $E_{c}$ and $E_{s}$ are no projections. But, from $E_{s}^{h} E_{s}=E_{s}, E_{s}^{h} R_{s}=R_{s}$ follows. Similarly $E_{c}^{h} R_{c}=R_{c}$ follows. Since $R_{s}(0)=\mathscr{Q}\left(\varepsilon^{2}\right)$ the ideas of the approximation theorem of [Sch92b] are not available at this point. An intermediate step is needed.

Lemma 11. Let $A_{0} \in H_{l, u}^{1}$ be an initial condition for the Ginzburg-Landau equation (2) with $\left\|A_{0}\right\|_{H_{l, u}^{1}}<C_{3}$ and $\tilde{u}_{0}$ an initial condition for (1). We write $K_{t}\left(\tilde{u}_{0}\right)$ as

$$
K_{t}\left(\tilde{u}_{0}\right)=\psi_{\varepsilon}\left(G_{\varepsilon^{2} t}\left(A_{0}\right)\right)+R_{c}(t)+R_{s}(t)
$$

with $R_{s}=E_{s}^{h} R_{s}$ and $R_{c}=E_{c}^{h} R_{c}$. Suppose $\left\|\left.R_{c}\right|_{t=0}\right\|_{H_{l, u}^{4}} \leq C_{4}^{\prime} \varepsilon^{3 / 2}$ and $\left\|\left.R_{s}\right|_{t=0}\right\|_{H_{l, u}^{4}} \leq$ $C_{4} \varepsilon^{5 / 4}$. Then there exists $C_{5}, C_{6}, \varepsilon_{0}>0$, only depending on $C_{3}, C_{4}$, and $C_{4}^{\prime}$, such that for all $0<\varepsilon<\varepsilon_{0}$, the following estimates are valid:
a) $\sup _{0 \leq t \leq 1 / \varepsilon^{1 / 4}}\left(\left\|R_{c}\right\|_{H_{l, u}^{4}}+\left\|R_{s}\right\|_{H_{l, u}^{4}}\right) \leq C_{5} \varepsilon^{5 / 4}$,
b) $\left\|\left.R_{s}\right|_{t=1 / \varepsilon^{1 / 4}}\right\|_{H_{l, u}^{4}} \leq C_{6} \varepsilon^{9 / 4}$.

At the end of this small approximation interval we are in a situation to prove the approximation property as in [Sch92b]. The only difference is that we only assume the initial conditions for the Ginzburg-Landau equation in $H_{l, u}^{1}$ instead of $H_{l, u}^{3}$. Contrary to previous work we also point out that the error $V_{-1} E_{1}^{h} R_{c}$ is in $H_{l, u}^{1, \varepsilon}$ instead of being only in $H_{l, u}^{4}$ at the end of the approximation interval. I.e it is a function of the slow space variable $X$. We use this to control the jumps $G_{T_{1}} A_{n}-A_{n+1}$. 
Lemma 12. Let $A_{0} \in H_{l, u}^{1}$ be an initial condition for the Ginzburg-Landau equation (2) and $\tilde{u}_{0}$ be an initial condition of (1). We write $K_{t}\left(\tilde{u}_{0}\right)$ as

$$
K_{t}\left(\tilde{u}_{0}\right)=\psi_{\varepsilon}\left(G_{\varepsilon^{2} t}\left(A_{0}\right)\right)+R_{c}(t)+R_{s}(t)
$$

with $R_{s}=E_{s}^{h} R_{s}$ and $R_{c}=E_{c}^{h} R_{c}$. Assume $\left\|\left.R_{c}\right|_{t=0}\right\|_{H_{l, u}^{4}} \leq C_{5} \varepsilon^{5 / 4}$ and $\left\|\left.R_{s}\right|_{t=0}\right\|_{H_{l, u}^{4}}<$ $C_{6} \varepsilon^{9 / 4}$. Then for all $T_{2}>0$ there exists $C_{s}, C_{c}, \varepsilon_{0}>0$, only depending on $T_{2}$, $\left\|A_{0}\right\|_{H_{l, u}^{1}}, C_{5}$, and $C_{6}$, such that for all $0<\varepsilon<\varepsilon_{0}$ the following estimates are valid:

a) $\sup _{0 \leq t \leq T_{2} / \varepsilon^{2}}\left\|R_{c}(t)\right\|_{H_{l, u}^{4}} \leq C_{c} \varepsilon^{5 / 4}$,

b) $\sup _{0 \leq t \leq T_{2} / \varepsilon^{2}}\left\|R_{s}(t)\right\|_{H_{l, u}^{4}} \leq C_{s} \varepsilon^{9 / 4}$,

c) $\left\|\left.V_{-1} E_{1}^{h} R_{c}\right|_{t=T_{2} / \varepsilon^{2}}\right\|_{H_{l, u}^{1, \varepsilon}} \leq C_{c} \varepsilon^{5 / 4}$.

By a combination of these lemmas and of Theorem 7 the main principles of the introduction can be shown.

(ATT), (APP) and (GL): The demands of the introduction can be fulfilled with $\beta=1 / 4, Z=H_{l, u}^{4}$, and $Y=H_{l, u}^{1}$. (GL) is given by Theorem 7, (ATT) is given by Lemma 10, and (APP) is given by a combination of Lemma 11 and Lemma 12. This proves Theorem 2 .

We remark that Lemma $12 \mathrm{c}$ ) is only needed to prove Theorem 3 . It is proved at the end of this section.

\subsection{Proof of the Lemmas}

To make the notation more general we write (1) as

$$
\partial_{t} u=\lambda\left(i \partial_{x}, \varepsilon^{2}\right) u+B(u, u),
$$

where $B(u, u)=\varrho\left(i \partial_{x}\right) u^{2}$ and $\lambda=-\left(1+\partial_{x}^{2}\right)^{2}+\varepsilon^{2}$. Before we prove these lemmas we have to do some work in advance.

\subsubsection{Properties of the Analytic Semigroup $e^{\lambda t}$}

To solve (1) we will use semigroup theory as it can be found in [Si85]. Since $\lambda$ is a sectorial operator in $L_{l, u}^{2}(\mathbb{R}, \mathbb{C})$ it generates an analytic semigroup $\left(e^{\lambda t}\right)_{t \geq 0}$ in this space. This semigroup can be represented by a multiplier $e^{\hat{\lambda}\left(k, \varepsilon^{2}\right) t}$ in Fourier space. We remark that by their definition with scalar multipliers the semigroup and the operators $E_{s}^{h}, E_{c}^{h}$ commute. These assertions can be shown with the help of multiplier theory and test functions. We summarize the later needed properties in the following lemma.

Lemma 13. There exists $\sigma>0$ such that for $0 \leq \varepsilon^{2} t \leq 1$ the following estimates are valid: 

a) $\left\|e^{\lambda t} E_{s}^{h} u_{0}\right\|_{H_{l, u}^{4}} \leq e^{-\sigma t}\left\|u_{0}\right\|_{H_{l, u}^{1}} \max \left(1, t^{-3 / 4}\right)$,
b) $\left\|\int_{0}^{t} e^{\lambda(t-\tau)} E_{s}^{h} u(\tau) d \tau\right\|_{H_{l, u}^{4}} \leq C \sup _{\tau \leq t}\|u(\tau)\|_{H_{l, u}^{1}}$,
c) $\left\|e^{\lambda t} E_{c}^{h} u_{0}\right\|_{H_{l, u}^{4}} \leq C\left\|u_{0}\right\|_{L_{l, u}^{2}}$,
d) $\left\|\varepsilon^{2} \int_{0}^{t} e^{\lambda(t-\tau)} E_{c}^{h} u(\tau) d \tau\right\|_{H_{l, u}^{4}} \leq C \varepsilon^{2} t \sup _{\tau \leq t}\|u(\tau)\|_{L_{l, u}^{2}}$,
e) $\left\|\left.\left(V_{-1} e^{\lambda t} E_{1}^{h} u_{0}\right)\right|_{t=1 / \varepsilon^{2}}\right\|_{H_{l, u}^{1, \varepsilon}} \leq C\left\|u_{0}\right\|_{L_{l, u}^{2}}$,
f) $\left\|\varepsilon^{2} V_{-1} \int_{0}^{t=1 / \varepsilon^{2}} e^{\lambda(t-\tau)} E_{1}^{h} u(\tau) d \tau\right\|_{H_{l, u}^{1, \varepsilon}} \leq C \sup _{\tau \leq t}\|u(\tau)\|_{L_{l, u}^{2}}$.

Proof. With help of Lemma 4 and Lemma 5 we have the following estimates:

a) $\left\|e^{\lambda t} E_{s}^{h} u_{0}\right\|_{H_{l, u}^{4}} \leq C\left\|e^{\hat{\lambda}\left(k, \varepsilon^{2}\right) t} \hat{E}_{s}^{h}(k)\left(1+|k|^{2}\right)^{3 / 2}\right\|_{C_{b}^{2}}\left\|u_{0}\right\|_{H_{l, u}^{1}}$,

b) $\left\|\int_{0}^{t} e^{\lambda(t-\tau)} E_{s}^{h} u(\tau) d \tau\right\|_{H_{l, u}^{4}}$

$$
\leq C \int_{0}^{t}\left\|e^{\hat{\lambda}\left(k, \varepsilon^{2}\right)(t-\tau)} \hat{E}_{s}^{h}(k)\left(1+|k|^{2}\right)^{3 / 2}\right\|_{C_{b}^{2}} d \tau \sup _{\tau \leq t}\|u(\tau)\|_{H_{l, u}^{1}},
$$

e) $\left\|\left.\left(V_{-1} e^{\lambda t} E_{1}^{h} u_{0}\right)\right|_{t=1 / \varepsilon^{2}}\right\|_{H_{l, u}^{1, \varepsilon}}$

$$
\leq C\left\|\left.e^{\hat{\lambda}\left(\varepsilon k, \varepsilon^{2}\right) t} \hat{E}_{1}^{h}(\varepsilon k)\left(1+\left|\frac{k-1}{\varepsilon}\right|^{2}\right)^{1 / 2}\right|_{t=1 / \varepsilon^{2}}\right\|_{C_{b}^{2}}\left\|u_{0}\right\|_{L_{l, u}^{2, \varepsilon}},
$$

f) $\left\|\varepsilon^{2} V_{-1} \int_{0}^{t=1 / \varepsilon^{2}} e^{\lambda(t-\tau)} E_{1}^{h} u(\tau) d \tau\right\|_{H_{l, u}^{1, \varepsilon}}$

$$
\leq \varepsilon^{2} C \int_{0}^{t=1 / \varepsilon^{2}}\left\|e^{\hat{\lambda}\left(\varepsilon k, \varepsilon^{2}\right)(t-\tau)} \hat{E}_{1}^{h}(\varepsilon k)\left(1+\left|\frac{k-1}{\varepsilon}\right|^{2}\right)^{1 / 2}\right\|_{C_{b}^{2}} d \tau \sup _{\tau \leq t}\|u\|_{L_{l, u}^{2, \varepsilon}} .
$$

Since $\|u\|_{L_{l, u}^{2, \varepsilon}} \leq C\|u\|_{L_{l, u}^{2}}$, it remains to estimate the norm of the multipliers. We will only estimate them in the $C_{b}^{0}$-norm to make the ideas clear. The derivatives can be estimated in the same manner. Due to the remark at the end of Sect. 2.1 the semigroup is exponentially damped in a) and b) and it acts on scaled functions in e) and $\mathrm{f}$ ).

For a) we get

$$
\begin{aligned}
\sup _{k}\left|e^{\hat{\lambda}\left(k, \varepsilon^{2}\right) t} \hat{E}_{s}^{h}(k)\left(1+|k|^{2}\right)^{3 / 2}\right| & \leq C \sup _{k}\left|e^{-\sigma t} e^{-c k^{4} t}(1+|k|)^{3}\right| \\
& \leq C e^{-\sigma t} \max \left(1, t^{-3 / 4}\right),
\end{aligned}
$$

due to the fact that $E_{s}^{h}$ extracts only uncritical modes. 
To estimate b) we look at

$$
\int_{0}^{t} \sup _{k}\left|e^{\hat{\lambda}\left(k, \varepsilon^{2}\right)(t-\tau)} \hat{E}_{s}^{h}(k)\left(1+|k|^{2}\right)^{3 / 2}\right| d \tau \leq \int_{0}^{t} C e^{-\sigma \tau} \max \left(1, \tau^{-3 / 4}\right) d \tau \leq C .
$$

This semigroup formates peaks in Fourier space. So we can show e),

$$
\begin{aligned}
& \sup _{k}\left|e^{\hat{\lambda}\left(k, \varepsilon^{2}\right) t} \hat{E}_{1}^{h}(k)\left(1+\left|\frac{k-1}{\varepsilon}\right|^{2}\right)^{1 / 2}\right| \\
& \leq\left. C \sup _{|k|<1 / 2}\left|e^{-k^{2} t}\right|_{t=1 / \varepsilon^{2}}\left|+C \sup _{|k|<1 / 2}\right| e^{-k^{2} t}\left|\frac{k}{\varepsilon}\right|\right|_{t=1 / \varepsilon^{2}} \mid \leq C,
\end{aligned}
$$

and $\mathrm{f}$ )

$$
\begin{aligned}
& \varepsilon^{2} \int_{0}^{t=1 / \varepsilon^{2}} \sup _{k}\left|e^{\hat{\lambda}\left(k, \varepsilon^{2}\right)(t-\tau)} \hat{E}_{1}^{h}(k)\left(1+\left|\frac{k-1}{\varepsilon}\right|^{2}\right)^{1 / 2}\right| d \tau \\
& \leq \varepsilon^{2} C \int_{0}^{t=1 / \varepsilon^{2}} \sup _{|k|<1 / 2}\left|e^{-k^{2} \tau}\right| d \tau+C \varepsilon^{2} \int_{0}^{t=1 / \varepsilon^{2}} \sup _{|k|<1 / 2}\left|e^{-k^{2}(t-\tau)}\right| \frac{k}{\varepsilon}|| d \tau \leq C .
\end{aligned}
$$

For showing c) and d) we define the multiplier $\tilde{E}$ by $k \mapsto \hat{\phi}_{0}(k / 15)$ in Fourier space. Since $\left\|\tilde{E} E_{c}^{h} u\right\|_{H_{l, u}^{4}} \leq C\left\|k \mapsto \hat{\phi}_{0}(k / 15)\left(1+|k|^{2}\right)^{-2}\right\|_{C_{b}^{2}}\left\|E_{c}^{h} u\right\|_{L_{l, u}^{2}}$ the rest of the proof of c) and d) is clear if we use the second estimate in Lemma 4.

\subsubsection{Proof of Lemma 10}

In a first step we show that the solution $K_{t}\left(u_{0}\right)$ filtered by $E_{s}^{h}$ is of order $\mathscr{O}\left(\varepsilon^{2}\right)$ after a very short time $1 / \varepsilon^{1 / 4}$. Starting again, now with this configuration it is shown in a second step that the solution filtered by $E_{1}^{h}$ is in $H_{l, u}^{1, \varepsilon}$ for a time $t=T_{0} / \varepsilon^{2}$.

Assume now an initial condition $u_{0}$ with $\left\|u_{0}\right\|_{H_{l, u}^{4}} \leq R_{0} \varepsilon$.

1st step: a) We set $v_{1}(\cdot, 0)=\frac{1}{\varepsilon} E_{c} u_{0}$ and $v_{2}(\cdot, 0)=\frac{1}{\varepsilon} E_{s} u_{0}$. Inserting $u=\varepsilon v_{1}+\varepsilon v_{2}$ in (8) we obtain

$$
\begin{aligned}
& v_{1}=\left.e^{\lambda t} v_{1}\right|_{t=0}+\varepsilon E_{c} \int e^{\lambda(t-s)} B\left(v_{1}+v_{2}, v_{1}+v_{2}\right) d s=: F_{1}\left(v_{1}, v_{2}\right), \\
& v_{2}=\left.e^{\lambda t} v_{2}\right|_{t=0}+\varepsilon E_{s} \int e^{\lambda(t-s)} B\left(v_{1}+v_{2}, v_{1}+v_{2}\right) d s=: F_{2}\left(v_{1}, v_{2}\right) .
\end{aligned}
$$

We remark that there is no need for separating the system in this way, since $E_{c}$ and $E_{s}$ are no projections. For $\varepsilon$ sufficiently small $F=\left(F_{1}, F_{2}\right)$ is a contraction in a ball with center $\left(\left.e^{\lambda t} v_{1}\right|_{t=0},\left.e^{\lambda t} v_{2}\right|_{t=0}\right)$ and radius of order $\odot\left(\varepsilon^{1 / 2}\right)$ in $C_{0}^{b}\left(\left[0,1 / \varepsilon^{1 / 4}\right], H_{l, u}^{4} \times\right.$ $H_{l, u}^{4}$ ). Therefore, $F$ has a fixed point and this system possesses a solution in this space with $\sup _{t \in\left[0,1 / \varepsilon^{1 / 4}\right]}\left\|v_{i}\right\|_{H_{l, u}^{4}} \leq 2 R_{0}$ for $\varepsilon$ sufficiently small. 
b) For the time $t=\frac{1}{\varepsilon^{1 / 4}}$ we have

$$
\left\|\left.v_{1}\right|_{t=1 / \varepsilon^{1 / 4}}\right\|_{H_{l, u}^{4}} \leq C R_{0}+C \varepsilon^{3 / 4} R_{0}^{2} \leq \tilde{C}_{1}
$$

and

$$
\left\|\left.v_{2}\right|_{t=1 / \varepsilon^{1 / 4}}\right\|_{H_{l, u}^{4}} \leq C e^{-\sigma / \varepsilon^{1 / 4}} R_{0}+C \varepsilon R_{0}^{2} \leq \tilde{C}_{2} \varepsilon
$$

if $\varepsilon$ is sufficiently small, since the semigroup $e^{\lambda t}$ filtered by $E_{s}^{h}$ is exponentially damped. The constants $\tilde{C}_{1}, \tilde{C}_{2}$ depend only on $R_{0}$ and not on $u_{0}$ itself.

2nd step: a) In the next step we set $\left.w_{2}\right|_{t=0}=\left.\frac{1}{\varepsilon} v_{2}\right|_{t=1 / \varepsilon^{1 / 4}}$ and $\left.w_{1}\right|_{t=0}=\left.v_{1}\right|_{t=1 / \varepsilon^{1 / 4}}$. Inserting now $u=\varepsilon w_{1}+\varepsilon^{2} w_{2}$ into (8) we separate the resulting equation in

$$
\begin{aligned}
& w_{1}=\left.e^{\lambda t} w_{1}\right|_{t=0}+\varepsilon^{2} \int_{0}^{t} e^{\lambda(t-\tau)} E_{c}\left(2 B\left(w_{1}, w_{2}\right)+\varepsilon B\left(w_{2}, w_{2}\right)\right) d \tau, \\
& w_{2}=\left.e^{\lambda t} w_{2}\right|_{t=0}+\int_{0}^{t} e^{\lambda(t-\tau)} E_{s}\left(B\left(w_{1}, w_{1}\right)+2 \varepsilon B\left(w_{1}, w_{2}\right)+\varepsilon^{2} B\left(w_{2}, w_{2}\right)\right) d \tau .
\end{aligned}
$$

Remark that $E_{c}^{h} B\left(w_{1}, w_{1}\right)=0$ due to the supports in Fourier space (see [Sch92b]). Obviously this system possesses solutions locally in time. The $\mathscr{Q}(1)$-boundedness and the existence on a long time interval $\left[0, \tilde{T}_{0} / \varepsilon^{2}\right]$ with $\tilde{T}_{0}=\mathscr{Q}(1) \leq 1$ is not clear. We take $\tilde{T}_{0} \leq 1$ to have an upper bound for some estimates. To show the $\mathscr{Q}(1)-$ boundedness we set $S_{i}(t)=\sup _{s \leq t}\left\|w_{i}(s)\right\|_{H_{l, u}^{4}}$ for $i=1,2$ and recall that $T=\varepsilon^{2} t$. We obtain from the second equation

$$
S_{2}(t) \leq \sup _{s \leq t}\left\|\left.e^{\lambda s} w_{2}\right|_{t=0}\right\|_{H_{l, u}^{4}}+C S_{1}(t)^{2}+\varepsilon C\left(S_{1}(t) S_{2}(t)+\varepsilon S_{2}(t)^{2}\right)
$$

and from the first equation

$$
S_{1}(t) \leq \sup _{s \leq t}\left\|\left.e^{\lambda s} w_{1}\right|_{t=0}\right\|_{H_{l, u}^{4}}+\varepsilon^{2} C \int_{0}^{t}\left(S_{1}(\tau) S_{2}(\tau)+\varepsilon S_{2}(\tau)^{2}\right) d \tau .
$$

We look now for a $\tilde{T}_{0}=\mathscr{O}(1)>0$, such that $S_{1}\left(\tilde{T}_{0} / \varepsilon^{2}\right) \leq \tilde{C}_{3}$ and $S_{2}\left(\tilde{T}_{0} / \varepsilon^{2}\right) \leq \tilde{C}_{4}$ for some constants $\tilde{C}_{3}$ and $\tilde{C}_{4}$. Choose now

$$
\begin{aligned}
& \tilde{C}_{3}=4 \sup _{1 / \varepsilon^{2} \geq s}\left\|\left.e^{\lambda s} w_{1}\right|_{t=0}\right\|_{H_{l, u}^{4}}=\mathscr{O}(1), \\
& \tilde{C}_{4}=\sup _{1 / \varepsilon^{2} \geq s}\left\|\left.e^{\lambda s} w_{2}\right|_{t=0}\right\|_{H_{l, u}^{4}}+C \tilde{C}_{3}^{2}+1=\mathscr{O}(1),
\end{aligned}
$$

and $\varepsilon$ such that $\varepsilon C\left(\tilde{C}_{3} \tilde{C}_{4}+\varepsilon \tilde{C}_{4}^{2}\right)<1$. This gives

$$
\begin{aligned}
S_{1}(t) \leq & \sup _{t \geq s}\left\|\left.e^{\lambda s} w_{1}\right|_{t=0}\right\|_{H_{l, u}^{4}} \\
& +\varepsilon^{2} C \int_{0}^{t}\left(S_{1}(\tau)\left[\sup _{t \geq s}\left\|\left.e^{\lambda s} w_{2}\right|_{t=0}\right\|_{H_{l, u}^{4}}+C \tilde{C}_{3}^{2}+1\right]\right)+\varepsilon \tilde{C}_{4}^{2} d \tau,
\end{aligned}
$$


and by Gronwall's inequality

$$
S_{1} \leq\left[\sup _{t \geq s}\left\|\left.e^{\lambda s} w_{1}\right|_{t=0}\right\|_{H_{l, u}^{4}}+\varepsilon C \tilde{C}_{4}^{2}\right] e^{C\left(\tilde{C}_{4}+C \tilde{C}_{3}^{2}+1\right) T}
$$

We choose now $\varepsilon$ again smaller such that $\varepsilon C \tilde{C}_{4}^{2}<\tilde{C}_{3} / 4$. Then we choose $T=\tilde{T}_{0}=$ Q(1) such that

$$
e^{C\left(\tilde{C}_{4}+C \tilde{C}_{3}^{2}+1\right) \tilde{T}_{0}}<2
$$

and so $S_{1}<\tilde{C}_{3}$ as demanded and hence $S_{2} \leq \tilde{C}_{4}$ by construction. Therefore, we have Q(1)-bounded solutions in $C_{0}^{b}\left(\left[0, \tilde{T}_{0} / \varepsilon^{2}\right), H_{l, u}^{4} \times H_{l, u}^{4}\right)$. Again $\tilde{C}_{3}$ and $\tilde{C}_{4}$ depend only on $R_{0}$.

b) Using Lemma 13 to estimate the right-hand side of (9) we get for $t=\tilde{T}_{0} / \varepsilon^{2}$,

$$
\left\|\left.V_{-1} E_{1}^{h} w_{1}\right|_{t=\tilde{T}_{0} / \varepsilon^{2}}\right\|_{H_{l, u}^{1, \varepsilon}} \leq \tilde{C}_{5}
$$

with $\tilde{C}_{5}$ only depending on $R_{0}$. We set $T_{0}=\tilde{T}_{0}+\varepsilon^{7 / 4}, w_{c}=\left.w_{1}\right|_{t=\tilde{T}_{0} / \varepsilon^{2}}$ and $w_{s}=\left.w_{2}\right|_{t=\tilde{T}_{0} / \varepsilon^{2}}$.

\subsubsection{Estimates for the Residuum}

For later computations we need exact estimates for the residuum.

Lemma 14. Let $A \in C\left([0,1], H_{l, u}^{1}\right)$ be a solution of the Ginzburg-Landau equation. Then we have

$$
\begin{aligned}
& \text { a) } \sup _{0 \leq l \leq t / \varepsilon^{2}}\left\|\int_{0}^{l} e^{\lambda(t-\tau)} E_{c}^{h} \operatorname{Res}\left(\psi_{\varepsilon}(A)\right) d \tau\right\|_{H_{l, u}^{4}(\mathbb{R}, \mathbb{C})} \leq C_{\operatorname{Res}} \varepsilon^{3 / 2} t^{1 / 8} \leq C_{\operatorname{Res}} \varepsilon^{5 / 4}, \\
& \text { b) } \sup _{0 \leq t \leq 1 / \varepsilon^{2}}\left\|\int_{0}^{t} e^{\lambda(t-\tau)} E_{s}^{h} \operatorname{Res}\left(\psi_{\varepsilon}(A)\right) d \tau\right\|_{H_{l, u}^{4}(\mathbb{R}, \mathbb{C})} \leq \mathscr{O}\left(\varepsilon^{5 / 2}\right) \leq C_{\operatorname{Res}} \varepsilon^{9 / 4}, \\
& \text { c) }\left\|_{0}^{t=1 / \varepsilon^{2}} V_{-1} e^{\lambda(t-\tau)} E_{1}^{h} \operatorname{Res}\left(\psi_{\varepsilon}(A)\right) d \tau\right\|_{H_{l, u}^{1, \varepsilon}(\mathbb{R}, \mathbb{C})} \leq \mathscr{O}\left(\varepsilon^{3 / 2}\right) \leq C_{\mathrm{Res}} \varepsilon^{5 / 4} .
\end{aligned}
$$

Proof. By inserting $\psi_{\varepsilon}(A)$ into (1) we compute the residuum. We denote by $\delta_{j n}$ a term in front of $e^{\imath j x}$, that is $\operatorname{Res}\left(\psi_{\varepsilon}(A)\right)=\sum_{j=-4}^{4} \sum_{n=1}^{n_{j}} \delta_{j n} e^{i j x}$. By using the formulae

$$
\begin{aligned}
\lambda\left(-i \partial_{x}, 0\right) B(\varepsilon x) e^{i n x}= & -e^{i n x}\left[\left(1-n^{2}\right)^{2} B+4 \operatorname{\varepsilon in}\left(1-n^{2}\right) \partial_{X} B\right. \\
& \left.+\varepsilon^{2}\left(2-6 n^{2}\right) \partial_{X}^{2} B+\varepsilon^{3} 4 i n \partial_{X}^{3} B+\varepsilon^{4} \partial_{X}^{4} B\right], \\
2 \varrho\left(\partial_{x}\right) B(\varepsilon x) e^{i n x}= & e^{i n x}\left[i n B+\varepsilon \partial_{X} B\right],
\end{aligned}
$$


the abbreviations $E_{0}^{c}=1-E_{0}$, and the fact that the $A_{\text {j }}^{0}$ are solutions of the modulation equations (6) we get

$$
\begin{aligned}
\delta_{01} & =\varepsilon^{3} \partial_{X}\left(\left(E_{0}\left(A_{1}^{0}\right)\left(E_{0} A_{-1}^{0}\right)\right)\right. \\
\delta_{02} & =\varepsilon^{5} \partial_{X}\left(\left(E_{0} A_{2}^{0}\right)\left(E_{0} A_{-2}^{0}\right)\right) \\
\delta_{11} & =\left(-\varepsilon^{4} 4 i \partial_{X}^{3}-\varepsilon^{5} \partial_{X}^{4}\right) E_{0} A_{1}^{0} \\
\delta_{12} & =\varepsilon^{4} \partial_{X}\left(\left(E_{0} A_{2}^{0}\right)\left(E_{0} A_{-1}^{0}\right)\right) \\
\delta_{13} & =\varepsilon^{3} \partial_{T} E_{0}^{c} A_{1}^{0} \\
\delta_{14} & =-\left(\varepsilon^{3}+4 \varepsilon^{3} \partial_{X}^{2}\right) E_{0}^{c} A_{1}^{0} \\
\delta_{15} & =-\varepsilon^{3} i\left(\left(E_{0} A_{2}^{0}\right)\left(E_{0}^{c} A_{-1}^{0}\right)+\left(E_{0}^{c} A_{2}^{0}\right)\left(E_{0} A_{-1}^{0}\right)+\left(E_{0}^{c} A_{2}^{0}\right)\left(E_{0}^{c} A_{-1}^{0}\right)\right) \\
\delta_{21} & =-\varepsilon^{4} \partial_{T} E_{0} A_{2}^{0} \\
\delta_{22} & =-\varepsilon^{2}\left(-24 i \varepsilon \partial_{X}-22 \varepsilon^{2} \partial_{X}^{2}+8 i \varepsilon^{3} \partial_{X}^{3}+\varepsilon^{4} \partial_{X}^{4}\right) E_{0} A_{2}^{0} \\
\delta_{23} & =\varepsilon^{4} E_{0} A_{2}^{0} \\
\delta_{24} & =\varepsilon^{3} \frac{1}{2} \partial_{X}\left(\left(E_{0} A_{1}^{0}\right)\left(E_{0} A_{1}^{0}\right)\right) \\
\delta_{25} & =9 \varepsilon^{2} E_{0}^{c} A_{2}^{0} \\
\delta_{26} & =-\varepsilon^{2} i\left(2\left(E_{0} A_{1}^{0}\right)\left(E_{0}^{c} A_{1}^{0}\right)+\left(E_{0}^{c} A_{1}^{0}\right)\left(E_{0}^{c} A_{1}^{0}\right)\right) \\
\delta_{31} & =\varepsilon^{3}\left(3 i+\varepsilon \partial_{X}\right)\left(\left(E_{0} A_{1}^{0}\right)\left(E_{0} A_{2}^{0}\right)\right) \\
\delta_{41} & =\frac{1}{2} \varepsilon^{4}\left(4 i+\varepsilon \partial_{X}\right)\left(\left(E_{0} A_{2}^{0}\right)\left(E_{0} A_{2}^{0}\right)\right) \\
\delta_{-j n} & =\bar{\delta}_{j n} .
\end{aligned}
$$

Now the terms $\delta_{j n}$ are estimated in various norms. By using the smoothing properties of the semigroup $e^{\lambda t}$ generated by $\lambda$ and of the flow of the Ginzburg-Landau equation it is also possible to show that $S_{1 / \varepsilon} V_{-1} E_{1}^{h} R_{c}$ lies in $H_{l, u}^{1}$ at the end of the approximation interval, contrary to previous work.

We define another multiplier $\tilde{E}$ by $k \mapsto \hat{\phi}_{0}(k / 15)$ in Fourier space. By applying it on the terms of the lemma it is easily seen with help of Lemma 4 that it is sufficient to show the estimates only in $L_{l, u}^{2}$ instead of $H_{l, u}^{4}$, since the $\delta_{j n}$ have compact support in Fourier space by construction. To get the estimate b) we have to look at $\delta_{\text {gn }}$ with $j \neq 1$. For the estimates a) and c) we have to look at $\delta_{1 n}$.

We start with b). Except for the estimate of the time derivative $\delta_{21}$ it is sufficient that $E_{0} A_{j}^{0} \in C\left([0,1], H_{l, u}^{1, \varepsilon}\right)$. Since $H_{l, u}^{1, \varepsilon}$ is an algebra, the product of such terms is also in $H_{l, u}^{1, \varepsilon}$. To estimate these terms we get by Lemma 4,

$$
\begin{aligned}
\left\|\int_{0}^{t} e^{\lambda(t-\tau)} E_{s}^{h} \delta_{j n} d \tau\right\|_{L_{l, u}^{2}} & \leq C \int_{0}^{t} e^{-\sigma(t-\tau)} d \tau \sup _{t}\left\|\delta_{i j}\right\|_{L_{l, u}^{2}} \\
& \leq C \sup _{t}\left\|\delta_{j n}\right\|_{L_{l, u}^{2}} \leq C \varepsilon^{-1 / 2} \sup _{t}\left\|\delta_{j n}\right\|_{L_{l, u}^{2, \varepsilon}} .
\end{aligned}
$$

This can now be estimated by Lemma 5 with $q=0, s=1$ and with the succeeding Remark 6 with $m=s=1$ on different multipliers. In $\delta_{01}, \delta_{02}, \delta_{24}, \delta_{31}$, and in $\delta_{41}$ we take $\varrho$ minus its first Taylor coefficient. In $\delta_{26}, \delta_{25}$ we take $E_{0}^{c}$ as multiplier and in $\delta_{22}$ we take $\lambda$ minus its first Taylor coefficient. The estimate of $\delta_{23}$ is clear. Summarized 
we have $\sup _{t \in\left[0,1 / \varepsilon^{2}\right]}\left\|\delta_{j n}\right\|_{L_{l, u}^{2}}=\mathscr{O}\left(\varepsilon^{5 / 2}\right)$ for $j \neq 1$ or $(j, n) \neq(2,1)$. To estimate the time derivative $\delta_{21}$ we use that $T \mapsto \sqrt{T} \partial_{T} A_{2}^{0} \in C\left([0,1], H_{l, u}^{0}\right)$. Applying again Lemma 5 we have to estimate

$$
\left\|\int_{0}^{t} e^{\hat{\lambda}\left(k, \varepsilon^{2}\right)(t-\tau)} \hat{E}_{s}^{h}(k) \frac{1}{\left(\varepsilon^{2} \tau\right)^{1 / 2}} d \tau\right\|_{C_{b}^{2}} \leq C \varepsilon^{-1} \int_{0}^{t} e^{-\sigma(t-\tau)} \tau^{-1 / 2} d \tau \leq C / \varepsilon
$$

and therefore,

$$
\sup _{0 \leq t \leq 1 / \varepsilon^{2}}\left\|\int_{0}^{t} e^{\lambda(t-\tau)} E_{s}^{h} \delta_{21} d \tau\right\|_{H_{l, u}^{4}}=\mathscr{O}\left(\varepsilon^{5 / 2}\right) .
$$

To show a) more refined methods are necessary. The main idea is as follows. At the beginning of the considered time interval we have high enough powers of $\varepsilon$ because of Remark 6. At the end of the time interval where the error should be in $H_{l, u}^{1, \varepsilon}$ we can use the smoothing properties of the semigroup and of the Ginzburg-Landau equation to get high enough powers of $\varepsilon$.

By the used functional analytic set-up one loses $\mathscr{Q}\left(\varepsilon^{-1 / 2}\right)$ due to

$$
\|\tilde{E} u\|_{H_{l, u}^{4}} \leq C\left\|k \mapsto \hat{\phi}_{0}(k / 15)\left(1+|k|^{2}\right)^{-2}\right\|_{C_{b}^{2}}\|u\|_{L_{l, u}^{2}} \leq C \varepsilon^{-1 / 2}\|u\|_{L_{l, u}^{2, \varepsilon}} .
$$

To estimate $\delta_{11}$ we apply Lemma 5 with $q=0$ and $s=11 / 4$ to the multiplier $\lambda$. Since the multiplier $\lambda(1+k)$ minus its Taylor polynomial of order 2 can be estimated by at least $|k|^{11 / 4}$, and since $T \mapsto T^{7 / 8} A_{1}^{0} \in H_{l, u}^{11 / 4}$ we have to estimate

$$
\varepsilon \int_{0}^{t}\left\|\frac{e^{\hat{\lambda}(\varepsilon k)(t-\tau)} \hat{E}_{1}^{h}(\varepsilon k) \varepsilon^{11 / 4} k^{11 / 4}}{\left(1+|k|^{2}\right)^{11 / 4}}\right\|_{C_{b}^{2}}\left(\frac{C}{\left(\varepsilon^{2} \tau\right)^{7 / 8}}\right) d \tau
$$

Because of notational complexity we will only look to the sup-norm. The derivatives can be estimated in the same way, since the multiplier acts on scaled funtions. We get

$$
\begin{aligned}
& \varepsilon \int_{0}^{t} \sup _{|k|<1 / 2}\left|\frac{e^{k^{2}(t-s)}|k|^{11 / 4}}{\left(1+\left|\frac{k}{\varepsilon}\right|\right)^{11 / 4}} \frac{C}{\left(\varepsilon^{2} s\right)^{7 / 8}}\right| d s \\
& \leq \varepsilon^{-1} \int_{0}^{T} \sup _{K}\left|\frac{e^{-K^{2}(T-S)}|\varepsilon|^{11 / 4}|K|^{11 / 4}}{(1+|K|)^{11 / 4}} \frac{C}{(S)^{7 / 8}}\right| d S \\
& \leq \varepsilon^{7 / 4} \int_{0}^{T} \sup _{K}\left|e^{-K^{2}(T-S)} \frac{C}{(S)^{7 / 8}}\right| d S \\
& \leq \varepsilon^{7 / 4} \int_{0}^{T} \frac{C}{(S)^{7 / 8}} d S \leq C \varepsilon^{2} t^{1 / 8} .
\end{aligned}
$$


In the same way we estimate $\delta_{12}$ with $\varrho$ instead of $\lambda$. To estimate $\delta_{13}$ note that $T \mapsto T^{7 / 8} \partial_{T} A_{1}^{0} \in C\left([0,1], H_{l, u}^{3 / 4}\right)$. By applying Remark 6 to the multiplier $E_{0}^{c}$ we win another $\mathscr{Q}\left(\varepsilon^{3 / 4}\right)$. By the same computations as for $\delta_{11}$ this estimate follows, too. Since $T \mapsto T^{7 / 8} \delta_{14} \in C\left([0,1), H_{l, u}^{3 / 4, \varepsilon}\right)$ this is also true for $\delta_{14}$. To estimate $\delta_{15}$ note that $\left\|E_{0}^{c} A_{j}^{0}\right\|_{L_{l, u}^{2, \varepsilon}}=\mathscr{O}(\varepsilon),\left\|E_{0}^{*} A_{j}^{0} E_{0}^{c} A_{i}^{0}\right\|_{L_{l, u}^{2, \varepsilon}} \leq\left\|E_{0}^{c} A_{i}^{0}\right\|_{L_{l, u}^{2, \varepsilon}}\left\|E_{0}^{*} A_{j}^{0}\right\|_{H_{l, u}^{1, \varepsilon}}$, and $T \mapsto T^{1 / 2} E_{0}^{*} A_{j}^{0} E_{0}^{c} A_{i}^{0} \in C\left([0,1], H_{l, u}^{1, \varepsilon}\right)$. Since $\left\|E_{0}^{c} A_{i}^{0}\right\|_{L_{l, u}^{2, \varepsilon}}=\mathscr{O}(\varepsilon)$ the estimate of $\delta_{15}$ is no problem at all.

To prove c) we look on $\delta_{11}$ as an example. Here, we apply Lemma 5 directly with $q=1$ and $s=5 / 2$ without losing $\mathscr{Q}\left(\varepsilon^{-1 / 2}\right)$. Again only the sup-norm of the multiplier is estimated. We get

$$
\begin{aligned}
\varepsilon & \int_{0}^{t=1 / \varepsilon^{2}} \sup _{|k|<1 / 2}\left|\frac{e^{k^{2}(t-s)}|k|^{5 / 2}}{\left(1+\left|\frac{k}{\varepsilon}\right|\right)^{3 / 2}} \frac{C}{\left(\varepsilon^{2} s\right)^{3 / 4}}\right| d s \\
& \leq \varepsilon^{-1} \int_{0}^{T=1} \sup _{K}\left|\frac{e^{-K^{2}(T-S)}|\varepsilon|^{5 / 2}|K|^{5 / 2}}{(1+|K|)^{5 / 2-1}} \frac{C}{(S)^{3 / 4}}\right| d S \\
& \leq \varepsilon^{3 / 2} \int_{0}^{T=1} \sup _{K}\left|e^{-K^{2}(T-S)}(1+|K|)^{1} \frac{C}{(S)^{3 / 4}}\right| d S \\
& \leq C \varepsilon^{3 / 2} \int_{0}^{1}\left|\frac{1}{\sqrt{1-S}} \frac{1}{(S)^{3 / 4}}\right| d S \leq C \varepsilon^{3 / 2} .
\end{aligned}
$$

The other terms $\delta_{1 n}$ can be estimated similarly to a). We remark that this is the essential estimate to show Lemma $12 \mathrm{c}$ ). It is possible, since the singularities appear on different ends of the integration interval.

\subsection{Proof of Lemma 11}

In the small time interval at the beginning of the approximation the error $R_{s}$ decays rapidly as the solutions converge to the center manifold in case of discrete spectrum.

We denote constants coming from the semigroup by $C_{J}$, from the approximation by $C_{\psi}$ and from the residuum by $C_{\text {Res }}$. Inserting $u=\varepsilon^{5 / 4} \tilde{R}_{c}+\varepsilon^{5 / 4} \tilde{R}_{s}+\psi_{\varepsilon}$ into (8), where $\tilde{R}_{c}=E_{c}^{h} \tilde{R}_{c}, \tilde{R}_{s}=E_{h}^{s} \tilde{R}_{s}$ and $\tilde{R}=\tilde{R}_{c}+\tilde{R}_{s}$ we get the system

$$
\begin{aligned}
& \partial_{t} \tilde{R}_{c}=\lambda \tilde{R}_{c}+2 \varepsilon E_{c} B\left(\tilde{R}, \psi_{\varepsilon} / \varepsilon\right)+\varepsilon^{5 / 4} E_{c} B(\tilde{R}, \tilde{R})+\varepsilon^{-5 / 4} E_{c} \operatorname{Res}\left(\psi_{\varepsilon}\right), \\
& \partial_{t} \tilde{R}_{s}=\lambda \tilde{R}_{s}+2 \varepsilon E_{s} B\left(\tilde{R}, \psi_{\varepsilon} / \varepsilon\right)+\varepsilon^{5 / 4} E_{s} B(\tilde{R}, \tilde{R})+\varepsilon^{-5 / 4} E_{s} \operatorname{Res}\left(\psi_{\varepsilon}\right),
\end{aligned}
$$

with the initial condition $\left.\left(\tilde{R}_{c}, \tilde{R}_{s}\right)\right|_{t=0}=\left(\left.\varepsilon^{-5 / 4} R_{c}\right|_{t=0},\left.\varepsilon^{-5 / 4} R_{s}\right|_{t=0}\right)$. With Lemma 14 we get

$$
\left\|\varepsilon^{-5 / 4} \int_{0}^{t} e^{\lambda(t-\tau)} E_{c} \operatorname{Res}\left(\psi_{\varepsilon}\right) d \tau\right\|_{H_{l, u}^{4}} \leq C_{\text {Res }}
$$


and

$$
\left\|\varepsilon^{-5 / 4} \int_{0}^{t} e^{\lambda(t-\tau)} E_{s} \operatorname{Res}\left(\psi_{\varepsilon}\right) d \tau\right\|_{H_{l, u}^{4}} \leq C_{\operatorname{Res}} \varepsilon
$$

uniformly on the required time interval $\left[0,1 / \varepsilon^{1 / 4}\right]$. Using Lemma 13 and $\left\|\left.\tilde{R}_{c}\right|_{t=0}\right\|_{H_{l, u}^{4}} \leq C_{4}^{\prime} \varepsilon^{1 / 4}$ it is easily seen that this system possesses solutions in $C_{0}^{b}\left(\left[0,1 / \varepsilon^{1 / 4}\right], H_{l, u}^{4} \times H_{l, u}^{4}\right)$ with $\sup _{\left[0,1 / \varepsilon^{1 / 4}\right.}\left(\left\|\tilde{R}_{c}\right\|_{H_{l, u}^{4}}+\left\|\tilde{R}_{s}\right\|_{H_{l, u}^{4}}\right)<C_{5}$, where the constant $C_{5}$ only depends on $C_{3}, C_{4}$ and $C_{4}^{\prime}$. For $t=1 / \varepsilon^{1 / 4}$ we have $\left\|\left.\tilde{R}_{c}\right|_{t=1 / \varepsilon^{1 / 4}}\right\|_{H_{l, u}^{4}}<$ $C_{5}$. For $\left.\tilde{R}_{s}\right|_{t=1 / \varepsilon^{1 / 4}}$ we get by Lemma 13 ,

$$
\begin{aligned}
\left\|\tilde{R}_{s}\right\|_{t=1 / \varepsilon^{1 / 4}} \|_{H_{l, u}^{4}} & \leq C_{J} e^{-\sigma / \varepsilon^{1 / 4}} C_{4}+\varepsilon C_{J} C_{\psi} C_{5}+\varepsilon^{5 / 4} C_{J} C_{5}^{2}+\varepsilon C_{\mathrm{Res}} \\
& \leq \varepsilon\left(2+C_{J} C_{\psi} C_{5}+C_{\mathrm{Res}}\right)=: \varepsilon C_{6}
\end{aligned}
$$

if we choose $\varepsilon$ so small that $C_{J} C_{4} e^{-\sigma / \varepsilon^{1 / 4}} \leq \varepsilon$ and $\varepsilon^{1 / 4} C_{J} C_{5}^{2} \leq 1$.

\subsection{Proof of Lemma 12}

On the long time interval we can prove the approximation theorem similarly to the proof of the approximation theorem of [Sch92b]. To simplify notation we take $T_{2}=1$. A solution of (8) can now be written as

$$
u=\psi_{\varepsilon}+\tilde{R}=\varepsilon \psi_{c}+\varepsilon^{2} \psi_{s}+\varepsilon^{5 / 4} \tilde{R}_{c}+\varepsilon^{9 / 4} \tilde{R}_{s},
$$

where $\tilde{R}_{c}=E_{c}^{h} \tilde{R}_{c}$ and $\tilde{R}_{s}=E_{s}^{h} \tilde{R}_{s}$. Inserting, this in (8) gives the system

$$
\begin{aligned}
& \partial_{t} \tilde{R}_{c}=\lambda \tilde{R}_{c}+\varepsilon^{2} L_{c}(\tilde{R})+\varepsilon^{9 / 4} N_{c}(\tilde{R})+\varepsilon^{-5 / 4} E_{c} \operatorname{Res}\left(\psi_{\varepsilon}\right), \\
& \partial_{t} \tilde{R}_{s}=\lambda \tilde{R}_{s}+L_{s}\left(\tilde{R}_{c}\right)+\varepsilon^{1 / 4} N_{s}(\tilde{R})+\varepsilon^{-9 / 4} E_{s} \operatorname{Res}\left(\psi_{\varepsilon}\right),
\end{aligned}
$$

with the abbreviations

$$
\begin{aligned}
L_{c}(\tilde{R})= & E_{c}\left(2 B\left(\tilde{R}_{c}, \psi_{s}\right)+2 B\left(\tilde{R}_{s}, \psi_{c}\right)\right), \\
L_{s}\left(\tilde{R}_{c}\right)= & E_{s}\left(2 B\left(\tilde{R}_{c}, \psi_{c}\right)\right), \\
N_{c}(\tilde{R})= & E_{c}\left(B\left(\tilde{R}_{s}, 2 \tilde{R}_{c}+2 \varepsilon^{3 / 4} \psi_{s}+\varepsilon \tilde{R}_{s}\right)\right), \\
N_{s}(\tilde{R})= & E_{s}\left(2 \varepsilon^{3 / 4} B\left(\tilde{R}_{c}, \psi_{s}\right)+2 \varepsilon^{3 / 4} B\left(\tilde{R}_{s}, \psi_{c}\right)+B\left(\tilde{R}_{c}, \tilde{R}_{c}\right)\right. \\
& \left.+\varepsilon B\left(\tilde{R}_{s}, 2 \tilde{R}_{c}+2 \varepsilon^{3 / 4} \psi_{s}+\varepsilon \tilde{R}_{s}\right)\right) .
\end{aligned}
$$

This system is solved in $C_{0}^{b}\left(\left[0,1 / \varepsilon^{2}\right], H_{l, u}^{4} \times H_{l, u}^{4}\right)$. Since the system can be solved locally in time it remains to show the $\mathscr{Q}(1)$-boundedness of the solutions on the required time interval. We abbreviate $S_{c}(t)=\sup _{0 \leq \tau \leq t}\left\|\tilde{R}_{c}(\tau)\right\|_{H_{l, u}^{4}}$ and $S_{s}(t)=$ $\sup _{0 \leq \tau \leq t}\left\|\tilde{R}_{s}(\tau)\right\|_{H_{l, u}^{4}}$. Again we denote constants coming from the semigroup by $C_{J}$, from the approximation by $C_{\psi}$ and from the residuum by $C_{\mathrm{Res}}$. Writing the second equation in integral form and using Lemma 14 gives

$$
S_{s} \leq C_{J} C_{\psi} S_{c}+M_{1}
$$


with

$$
\begin{aligned}
& M_{1}=M_{2}+C_{\mathrm{Res}}+C_{J} C_{6}, \\
& M_{2}=\varepsilon^{1 / 4} C_{J}\left[\varepsilon^{3 / 4} C_{\psi} S_{c}+\varepsilon^{3 / 4} C_{\psi} S_{s}+S_{c}^{2}+\varepsilon S_{s}\left(S_{c}+\varepsilon^{3 / 4} C_{\psi}+\varepsilon S_{s}\right)\right] .
\end{aligned}
$$

The first equation gives

$$
S_{c} \leq \varepsilon^{2} S_{J} \int_{0}^{t}\left(C_{\psi} S_{c}(\tau)+C_{\psi} S_{s}(\tau)\right) d \tau+M_{3}
$$

with

$$
\begin{aligned}
& M_{3}=M_{4}+C_{\mathrm{Res}}+C_{5} C_{J} \\
& M_{4}=\varepsilon^{1 / 4} C_{J} S_{s}\left(S_{c}+\varepsilon^{3 / 4} C_{\psi}+\varepsilon S_{s}\right)
\end{aligned}
$$

Later on we choose $\varepsilon$ small enough such that $M_{i} \leq 1$ with $i=2,4$. By inserting the above expression for $S_{s}$ into that of $S_{c}$ we obtain

$$
\begin{aligned}
S_{c} & \leq M_{3}+\varepsilon^{2} \int C_{J}\left(C_{\psi} S_{c}+C_{\psi} C_{J} C_{\psi} S_{c}\right) d \tau+\varepsilon^{2} \int C_{J} C_{\psi} M_{1} d \tau \\
& \leq M_{3}+C_{J}^{2} C_{\psi} M_{1}+\varepsilon^{2} \int C_{J}\left(C_{\psi}+C_{\psi}^{2} C_{J}\right) S_{c} d \tau
\end{aligned}
$$

By Gronwall's inequality one gets

$$
\begin{aligned}
S_{c} & \leq\left(M_{3}+C_{J}^{2} C_{\psi} M_{1}\right) e^{C_{J}\left(C_{\psi}+C_{\psi}^{2} C_{J}\right) T} \\
& \leq\left(M_{3}+C_{J}^{2} C_{\psi} M_{1}\right) e^{C_{J}\left(C_{\psi}+C_{\psi}^{2} C_{J}\right)}=: C_{c} .
\end{aligned}
$$

Inserting this in (13) gives

$$
S_{s} \leq M_{1}+C_{J} C_{\psi} C_{c}=: C_{s}
$$

Next we choose $\varepsilon$ so small that

$$
\begin{aligned}
& M_{2} \leq \varepsilon^{1 / 4} C_{J}\left[\varepsilon^{3 / 4} C_{\psi} C_{c}+\varepsilon^{3 / 4} C_{\psi} C_{s}+C_{c}^{2}+\varepsilon C_{s}\left(C_{c}+\varepsilon^{3 / 4} C_{\psi}+\varepsilon C_{s}\right)\right] \leq 1 \\
& M_{4} \leq \varepsilon^{1 / 4} C_{J} C_{s}\left(C_{c}+\varepsilon^{3 / 4} C_{\psi}+\varepsilon C_{s}\right) \leq 1
\end{aligned}
$$

It remains to estimate $V^{-1} E_{h}^{1} \tilde{R}_{c} \in H_{l, u}^{1, \varepsilon}$ for $t=1 / \varepsilon^{2}$. By the above computations we have $\left\|L_{c}(\tilde{R})\right\|_{L_{l, u}^{2}} \leq C,\left\|N_{c}(\tilde{R})\right\|_{L_{l, u}^{2}} \leq C$, and $\left\|\left.\tilde{R}_{c}\right|_{t=0}\right\|_{L_{l, u}^{2}} \leq C$. Using also Lemma 14 we can apply Lemma 13 to the variation of constants formula which transforms (12) into an integral equation. There we estimate the right-hand side in $H_{l, u}^{1, \varepsilon}$ as in (9) and (10) and so the assertion follows.

If we would have inverted the linear part of (12) as has been done in former works, the estimates for the residuum would have been much more complicated. So we choose this way. 


\subsection{Proof of Theorem 3}

With help of these lemmas we start now to construct the pseudo-orbit of approximations. Using Lemma 10 we have constructed the first initial condition for the solution $A_{1}$ of our sequence of initial conditions for the Ginzburg-Landau equation (2) at a time $t=T_{0} / \varepsilon^{2}$. We solve the Ginzburg-Landau equation on the time interval $\left[T_{0}, T_{0}+T_{1}\right]$. The associated approximation is given by $\psi_{\varepsilon}\left(G_{\varepsilon^{2} t-T_{0}}\left(A_{1}\right)\right)$. On the time interval $\left[T_{0} / \varepsilon^{2}, T_{0} / \varepsilon^{2}+1 / \varepsilon^{1 / 4}\right)$ we use Lemma 11 as the approximation theorem. On the time interval $\left[T_{0}+1 / \varepsilon^{1 / 4},\left(T_{0}+T_{1}\right) / \varepsilon^{2}\right)$ we take Lemma 12 . The critical resp. the uncritical part of the error on the time interval $\left[T_{0} / \varepsilon^{2},\left(T_{0}+T_{1}\right) / \varepsilon^{2}\right]$ is denoted by $R_{c, 1}$ resp. $R_{s, 1}$. To get the new initial condition $A_{2}$ for the Ginzburg-Landau equation, we add the critical error $\left.\varepsilon^{-1} S_{1 / \varepsilon} V_{-1} E_{h}^{1} R_{c, 1}\right|_{t=\left(T_{0}+T_{1}\right) / \varepsilon^{2}} \in H_{l, u}^{1}$ to the actual solution $G_{T_{1}}\left(A_{1}\right)$ of the Ginzburg-Landau equation. By changing from $G_{T_{1}\left(A_{1}\right)}$ to $A_{2}$, the error $R_{c, 1}$ changes to $R_{c, 2}$ and the error $R_{s, 1}$ to $R_{s, 2}$. By construction we have

$$
\left.R_{c, 2}\right|_{t=\left(T_{0}+T_{1}\right) / \varepsilon^{2}}=\left.V_{1}(1-E) V_{-1} E_{h}^{1} R_{c, 1}\right|_{t=\left(T_{0}+T_{1}\right) / \varepsilon^{2}}+\text { c.c. }
$$

Therefore $\left\|\left.R_{c, 2}\right|_{t=\left(T_{0}+T_{1}\right) / \varepsilon^{2}}\right\|_{H_{l, u}^{4}} \leq C C_{c} \varepsilon^{7 / 4}$ as in (7). Hence we can apply Lemma 11 and Lemma 12 again and estimate the difference $\psi_{\varepsilon}\left(G_{\varepsilon^{2} t-\left(T_{0}+T_{1}\right)}\left(A_{2}\right)\right)-K_{t}\left(u_{0}\right)$ on the time interval $\left[\left(T_{0}+T_{1}\right) / \varepsilon^{2},\left(T_{0}+2 T_{1}\right) / \varepsilon^{2}\right]$. The sequence of initial conditions is defined inductively by

$$
A_{n}=G_{T_{0}+(n+1) T_{1}}\left(A_{n-1}\right)+\left.\varepsilon^{-1} S_{1 / \varepsilon} V_{-1} E_{h}^{1} R_{c, n-1}\right|_{t=\left(T_{0}+(n-1) T_{1}\right) / \varepsilon^{2}} .
$$

It remains to show that the constants of Theorem 3 are bounded uniformly in time. We show that the initial conditions $A_{n}$ and the errors $R_{s, n}, R_{c, n}$ are bounded uniformly in time. Since all constants can be controlled by $C_{3}, C_{4}$ and $C_{4}^{\prime}$ of Lemma 11, we will be finished. We first estimate the initial conditions $A_{n}$.

Lemma 15. There exists $\varepsilon_{0}>0$ such that for all $\varepsilon<\varepsilon_{0}$ we have

$$
\left\|A_{n}\right\|_{H_{l, u}^{1}} \leq \max \left\{3 C_{b},\left\|A_{1}\right\|_{H_{l, u}^{1}}\right\}=: C_{3} \text {. }
$$

Proof. Since $\left\|\left.\varepsilon^{-1} S_{1 / \varepsilon} V_{-1} E_{1}^{h} R_{c}\right|_{T=T_{0}+T_{1}}\right\|_{H_{l, u}^{1}} \leq C_{c} \varepsilon^{1 / 4}$, and since $\left\|G_{T_{1}}\left(A_{1}\right)\right\|_{H_{l, u}^{1}} \leq$ $C_{b}+e^{-1}\left\|A_{1}\right\|_{H_{l, u}^{1}}$ for $T_{1} \geq 1$, we have

$$
\left\|A_{2}\right\|_{H_{l, u}^{1}} \leq \varepsilon^{1 / 4} C_{c}+C_{b}+e^{-1}\left\|A_{1}\right\|_{H_{l, u}^{1}} \leq \max \left\{3 C_{b},\left\|A_{1}\right\|_{H_{l, u}^{1}}\right\}
$$

for $\varepsilon$ sufficiently small. For $n>2$ the assertion follows by induction.

For the uncritical part we obtain

Lemma 16. There exists $C_{7}, \varepsilon_{0}>0$ such that for all $0<\varepsilon<\varepsilon_{0}$ we have

$$
\left\|\left.R_{s, n+1}\right|_{t=\left(T_{0}+n T_{1}\right) / \varepsilon^{2}}\right\|_{H_{l, u}^{4}} \leq C_{7} \varepsilon^{9 / 4} \leq C_{4} \varepsilon^{5 / 4}
$$

with $C_{4}:=\max \left\{1,\left\|\left.\varepsilon^{-5 / 4} R_{s, 1}\right|_{t=T_{0} / \varepsilon^{2}}\right\|_{H_{l, u}^{4}}\right\}$.

Proof. We get

$$
\begin{aligned}
\left.R_{s, 2}\right|_{t=\left(T_{0}+T_{1}\right) / \varepsilon^{2}} & =\left.R_{s, 1}\right|_{t=\left(T_{0}+T_{1}\right) / \varepsilon^{2}}+\varepsilon^{2} \psi_{s}\left(G_{T_{1}}\left(A_{1}\right)\right)-\varepsilon^{2} \psi_{s}\left(A_{2}\right) \\
& =\left.R_{s, 1}\right|_{t=\left(T_{0}+T_{1}\right) / \varepsilon^{2}}+\frac{i}{9}\left(\varepsilon^{2} E_{0}\left(-A_{2}^{2}+\left(G_{T_{1}} A_{1}\right)^{2}\right) e^{2 \imath x}+\text { c.c. }\right),
\end{aligned}
$$


and so by Lemma 11 and Lemma 12,

$$
\begin{aligned}
\left\|\left.R_{s, 2}\right|_{t=\left(T_{0}+T_{1}\right) / \varepsilon^{2}}\right\|_{H_{l, u}^{4}} & \\
\leq & \left\|\left.R_{s, 1}\right|_{t=\left(T_{0}+T_{1}\right) / \varepsilon^{2}}\right\|_{H_{l, u}^{4}}+\varepsilon C\left(\left\|G_{T_{1}}\left(A_{1}\right)\right\|_{H_{l, u}^{1}}+\left\|A_{2}\right\|_{H_{l, u}^{1}}\right) \\
& \times\left\|\left.V_{-1} E_{1}^{h} R_{c, 1}\right|_{t=\left(T_{0}+T_{1}\right) / \varepsilon^{2}}\right\|_{H_{l, u}^{1, \varepsilon}} \\
\leq & \varepsilon^{9 / 4} C_{s}+\varepsilon^{9 / 4} C C_{c} \leq C_{7} \varepsilon^{9 / 4} .
\end{aligned}
$$

For $n>2$ the assertion follows by induction.

For the critical part we obtain

Lemma 17. There exists $C_{8}, \varepsilon_{0}>0$ such that for all $0<\varepsilon<\varepsilon_{0}$ we have

$$
\left\|\left.R_{c, n+1}\right|_{t=\left(T_{0}+n T_{1}\right) / \varepsilon^{2}}\right\|_{H_{l, u}^{4}} \leq C_{8} \varepsilon^{3 / 2}
$$

with $C_{8}:=\max \left\{1,\left\|\left.\varepsilon^{-6 / 4} R_{c, 1}\right|_{T=T_{0} / \varepsilon^{2}}\right\|_{H_{l, u}^{4}}\right\}$.

Proof. We have already obtained

$$
\begin{aligned}
\left\|\left.R_{c, 2}\right|_{t=\left(T_{0}+T_{1}\right) / \varepsilon^{2}}\right\|_{H_{l, u}^{4}} & \leq C \varepsilon^{1 / 2}\left\|\left.R_{c, 1}\right|_{t=\left(T_{0}+T_{1}\right) / \varepsilon^{2}}\right\|_{H_{l, u}^{4}} \\
& \leq C \varepsilon^{1 / 2} C_{c} \varepsilon^{5 / 4} \leq C C_{c} \varepsilon^{7 / 4} \leq C_{8} \varepsilon^{3 / 2}
\end{aligned}
$$

for $\varepsilon$ sufficiently small. For $n>2$ the assertion follows by induction.

Now we have constructed the pseudo-orbit $\psi$ with the required properties of Theorem 3 and so this theorem is proved. Since all constants are bounded uniformly, the pseudo-orbit stays bounded in $H_{l, u}^{4}$ and so the shadowed solution, too.

\section{Discussions}

It is possible to make the error smaller in powers of $\varepsilon$ by taking more terms into the approximation. To do this one has to include more peaks in Fourier space into the analysis. As in [Eck93] the larger peaks have to be chosen more regular than the smaller ones. So various approximation theorems and various theorems for attractivity are possible. Also various combinations of these theorems are possible to prove global existence of solutions of the original system.

It is clear that the above conclusions can also be drawn for every other exponentially attracting set of the Ginzburg-Landau equation. Also diffusive stability can be sufficient to apply the above theory. It is a subject of further research to apply this method to concrete sets of functions. It can be applied in the spatial periodic case, especially with very large spatial period, where more or less complicated attractors are known.

Using the whole functional analytical set-up of [Sch93] allows to generalize this method to situations where $u(x)$ is vectorvalued, e.g. has values in a Hilbert space. In fact this allows us the handling of Bénard's problem or of Taylor-Couette problem. This will be the subject of a forthcombing paper. 


\section{References}

[CE90] Collet, P., Eckmann, J.-P.: The time dependent amplitude equation for the SwiftHohenberg problem. Commun. Math. Phys. 132, 139-153 (1990)

[diPES71] di Prima, R.C., Eckhaus, W., Segel, L.A.: Nonlinear wave-number interaction in nearcritical two-dimensional flows. J. Fluid. Mech. 49, 705-744 (1971)

[Eck93] Eckhaus, W.: The Ginzburg-Landau equation is an attractor. J. Nonlinear Sci. 3, 329-348 (1993)

[He81] Henry, D.: Geometric theory of semilinear parabolic equations. LNM 840, Berlin, Heidelberg, New York: Springer 1981

[Ho85] Hörmander, L.: The analysis of linear partial differential operators. Berlin, Heidelberg, New York: Springer 1983-1985

[IMD89] Iooss, G., Mielke, A., Demay, Y.: Theory of steady Ginzburg-Landau equation, in hydrodynamic stability problems. Eur. J. Mech., B/Fluids, 8, nr. 3, 229-268 (1989)

[KSM92] Kirrmann, P., Schneider, G., Mielke, A.: The validity of modulation equations for extended systems with cubic nonlinearities. Proc. Roy. Soc. Edinburgh 122A, 85-91 (1992)

[LM72] Lions, J.L., Magenes, E.: Non-Homogeneous Boundary Value Problems and Applications. Berlin, Heidelberg, New York: Springer 1972

[Mi92] Mielke, A.: Reduction of PDE's on domains with several unbounded directions. J. Appl. Math. Phys. (ZAMP) 43 (1992)

[NW69] Newell, A., Whitehead, J.: Finite bandwidth, finite amplitude convection. J. Fluid Mech. 38, 279-303 (1969)

[Sch92a] Schneider, G.: Die Gültigkeit der Ginzburg-Landau-Approximation. Dissertation, Universität Stuttgart, 1992

[Sch92b] Schneider, G.: A new estimate for the Ginzburg-Landau approximation on the real axis. J. Nonlinear Sci., to appear

[Sch93] Schneider, G.: Error estimates for the Ginzburg-Landau approximation. ZAMP, to appear

[Si85] Sinestrari, E.: On the abstract Cauchy Problem of Parabolic Type in Spaces of Continuous Functions. J. Math. Anal. and Appl. 107, 16-66 (1985)

[vH91] van Harten, A.: On the validity of Ginzburg-Landau's equation. J. Nonlinear Sci. 1, $397-422(1991)$

[vH92] van Harten, A.: Decay to the Ginzburg-Landau manifold. Lecture held in Stuttgart, 1992 
